\title{
Construction of Novel Prognostic Nomogram for Mucinous and Signet Ring Cell Colorectal Cancer Patients with a Survival Longer Than 5 Years
}

\author{
Juan $X u^{1,2, *}$, Ziwei Sun $\mathbb{D}^{1,2, *}$, Huanyu Ju ${ }^{1,2}$, Erfu $X i^{1,2}$, Yuan $M u^{1,2}$, Jian $X u \mathbb{D}^{1,2}$, Shiyang Pan ${ }^{1,2}$ \\ 'Department of Laboratory Medicine, The First Affiliated Hospital of Nanjing Medical University, Nanjing, People's Republic of China; ${ }^{2}$ Branch of \\ National Clinical Research Center for Laboratory Medicine, Nanjing, People's Republic of China
}

*These authors contributed equally to this work

Correspondence: Shiyang Pan, Tel +86 I39 5I8I 4639, Email sypan@njmu.edu.cn

\begin{abstract}
Purpose: Mucinous adenocarcinoma (MA) and signet ring cell carcinoma (SRCC) are aggressive colorectal cancer histological subtypes with dismal prognosis. This study investigated prognostic factors and constructed novel nomograms for MA and SRCC patients who survived for over 5 years to optimize the follow-up regime, especially for early-onset patients.

Patients and Methods: Data from the Surveillance, Epidemiology, and End Results (SEER) database registered between 2004 and 2018 were extracted. MA and SRCC patients were divided into two groups with survival time of 5 years as a cut-off point. Prognostic factors for overall survival (OS) and cancer-specific survival (CSS) were determined by Cox regression models, and survival curves were plotted by the Kaplan-Meier method.

Results: We identified 8286 MA patients (45.73\%) and 551 SRCC patients (20.32\%) who survived for over 5 years. Multivariable Cox analyses identified age, tumor location, $\mathrm{N}$ stage, metastasis, CEA level, surgery, and lymph nodes dissection as independent risk factors for MACSS. SRCC was more aggressive and only N2 stage $(P=0.011)$ and metastasis $(P=0.043)$ were inversely associated with SRCCSS. Furthermore, we observed that small tumor size, well differentiation, and chemotherapy no longer provided survival benefit to $\geq 5$-year survivors. Therefore, we constructed novel nomograms appropriate for MA patients who survived for over 5 years. The consistency indexes for predicting 10-year OS and CSS were respectively $0.717,0.712$ in the training cohort and $0.727,0.735$ in the validation cohort. Conclusion: Our well-calibrated nomograms represent the first clinical prognostic models developed especially for MA patients with a survival longer than 5 years. For both MA and SRCC patients, TNM stage was a stable prognostic factor, while the prognostic values of tumor size, differentiation grade, and chemotherapy changed over time. We are hopeful that our prognostic models will help define personalized follow-up managements to further prolong patient survival.
\end{abstract}

Keywords: mucinous and signet ring cell colorectal cancer, survival over 5 years, changes in prognostic factors, nomogram, chemotherapy, SEER

\section{Introduction}

Worldwide, colorectal cancer (CRC) is the third most common cause of cancer morbidity, and ranks second in terms of mortality. ${ }^{1,2}$ In 2020, CRC accounted for more than 1.9 million new cancer cases and approximately 0.9 million deaths globally. Global estimates for 2020 also indicated that the age-standardized incidence of CRC was $44 \%$ higher in men than in women and 4-fold higher in countries with a higher Human Development Index than in transitioning countries. ${ }^{3}$ Since the early 2000s, a rapid decline (by 3\% annually) in CRC incidence, attributed to increased colonoscopy screening and removal of precursor lesions, has been observed in the elderly population. ${ }^{4}$ However, the reduction in new CRC cases in adults aged $\geq 50$ years is counterbalanced by increasing rates (by $1 \%$ to $4 \%$ per year) of early-onset CRC (age at diagnosis $<50$ years) ${ }^{5}$ 
Mucinous adenocarcinoma (MA) and signet ring cell carcinoma (SRCC) are both rare histological subtypes of CRC and have distinct clinicopathological and genetic features. ${ }^{6,7}$ Approximately $10-20 \%$ of CRC patients exhibit the MA subtype, where extracellular mucin comprises more than $50 \%$ of the tumor area. ${ }^{8,9}$ In turn, SRCC accounts for $1 \%$ of $\mathrm{CRC}$ cases and is characterized by abundant intracytoplasmic mucin and signet-ring cells making up more than $50 \%$ of the tumor volume. ${ }^{7,10}$ Though the prognosis of MA is still controversial, SRCC is considered highly aggressive and associated with worse prognosis.

At present, since no clinical guidelines have been specifically developed, the standard treatments for colorectal adenocarcinoma are recommended for MA and SRCC patients. ${ }^{11}$ Importantly, traditional prognostic factors for MA and SRCC were identified based on 5-year survival rates, and prognostic nomograms proposed so far were mostly used to predict only 5-year survival probability. In light of this, and considering that the prognostic value of baseline clinical factors may shift over time, there is a clear need to develop robust prognostic models for the large number of MA and SRCC patients who survived longer than 5 years. Research focusing on developing novel predictive nomograms for longer-term MA and SRCC survivors will provide supporting evidence for developing personalized management strategy to reduce CRC recurrence and mortality. Moreover, identifying predictors of long-term survival is expected to have substantial implications not only for clinical practice, but also for patient counseling and for research on the mechanisms of late metastasis.

Accordingly, the aim of this study was to investigate the clinicopathological characteristics and treatments of MA and SRCC patients who survived for over 5 years, in order to identify reliable prognostic factors to assist clinical treatment decisions. To this end, we obtained the most recent population-based CRC data contained in the Surveillance, Epidemiology, and End Results (SEER) database, whose large sized, diverse patient population allows for the investigation of clinical predictors for MA and SRCC patients. Moreover, we developed and validated novel nomograms to predict 10-year overall and cancer-specific survival probability in this group of patients. We believe that the nomograms provided here will be valuable to optimize the follow-up regime and improve long-term survival, especially for earlyonset patients.

\section{Materials and Methods Study Population}

We performed a retrospective analysis using the Surveillance, Epidemiology, and End Results (SEER) database (National Cancer Institute, NIH, Bethesda, MD, USA), which collects and reports authoritative information about clinicopathological features, cancer incidence, mortality, and survival in the United States. All cancer patients from 18 population-based cancer registries were enrolled. The November 2018 SEER data submission was queried by SEER*Stat software, version 8.3.2, and all patients with CRC registered from 2004 to 2018 were included in our study population. We have provided a signed SEER research data agreement form to the SEER program to obtain approval to access and analyze the SEER database. Since the SEER database is available to the public, ethical approval by the NIH Office of Human Subjects Research was not required.

\section{Cohort Selection}

Patients with CRC (C20.9) diagnosed after 2004 were selected for this study according to the International Classification of Diseases for Oncology codes (3rd edition, ICD-O-3), and CRC histological subtypes were identified as SRCC (code 8490 ) and MA (codes 8480 and 8481). The diagnosis was confirmed by positive histology at pathological analysis. The following exclusion criteria were applied: (I) Patients with more than one primary tumor; (II) Patients with less than 5-year documented survival time due to termination of follow-up; (III) Patients with survival time unknown or less than 1 month; (IV) Patients with no prognostic data or no surgery information; (V) Patients with primary tumor located in the appendix or large intestine. The flowchart of case selection is presented in Figure 1. To analyze the clinicopathological characteristics of the patients included in the study, they were divided into two groups: (1) Survival time $<5$ years $(\mathrm{ST}<5 \mathrm{Y}$ ), and (2) Survival time $\geq 5$ years ( $\mathrm{ST} \geq 5 \mathrm{Y}$ ). The latter group of patients was mainly diagnosed from 2004-2013, whereas patients with survival time $<5$ years were diagnosed from 2004 to 2018 . 


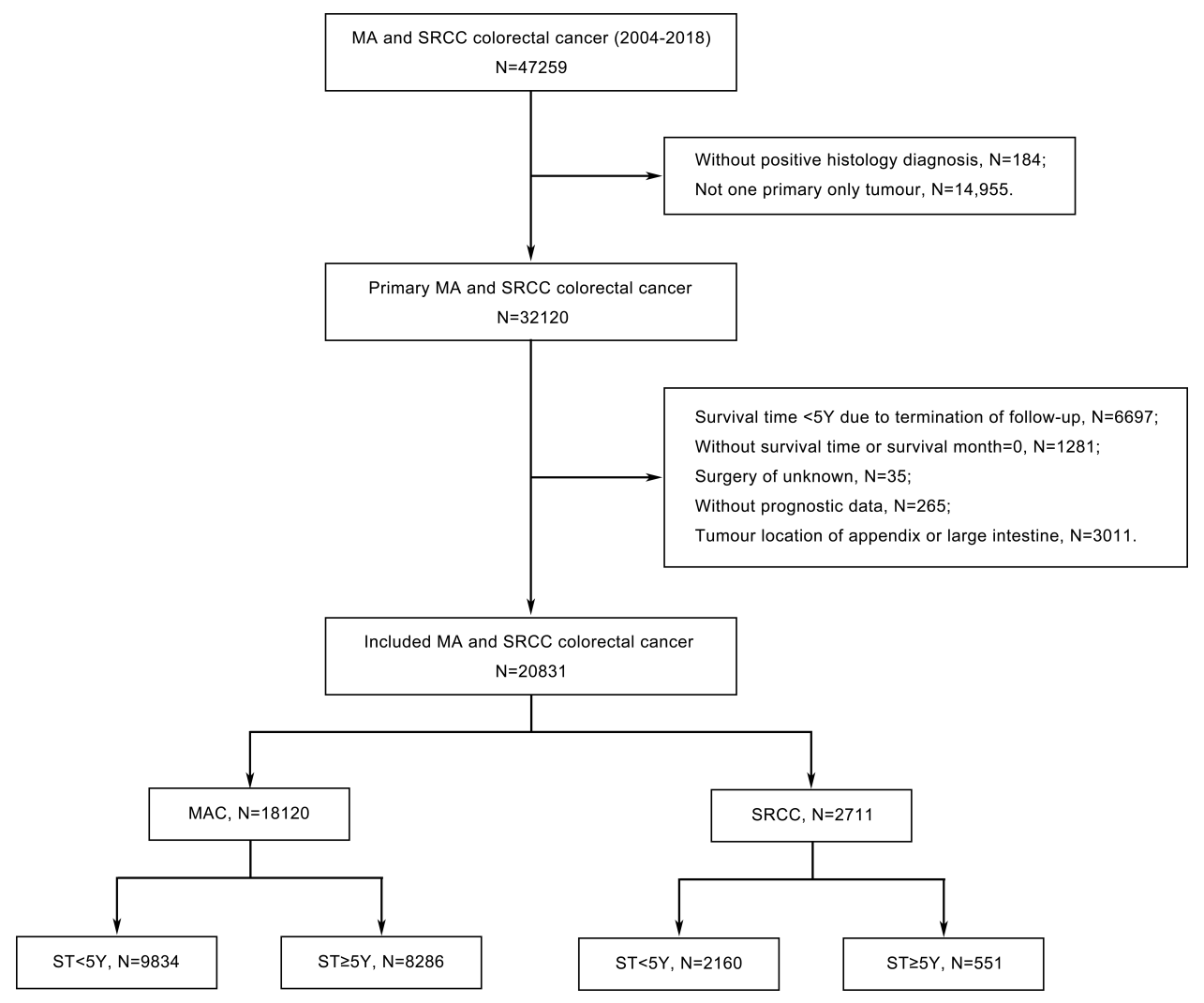

Figure I Flow chart for the creation of the included MA and SRCC patient cohort.

Abbreviations: MA, mucinous adenocarcinoma; SRCC, signet ring cell carcinoma; ST, survival time; Y, year.

\section{Outcome Measurement}

We collected the following variables for each case: age, gender, race, marital status, primary tumor site, tumor size, tumor grade, American Joint Committee on Cancer (AJCC) stage, carcinoembryonic antigen (CEA) level, tumor deposit, perineural invasion, surgery, lymph nodes dissection, chemotherapy, radiotherapy, systemic therapy, and survival outcome. Overall survival (OS) and cause-specific survival (CSS) were the main endpoints. OS was defined as the time from the date of diagnosis to the date of death due to any cause or until the date of last follow-up. CSS was defined by specifying colon cancer as the cause of death, measured from time of diagnosis until cancer-associated death or the end of follow up. Baseline characteristics were assessed to determine whether there were significant differences in the distribution of the study population into $\mathrm{ST}<5 \mathrm{Y}$ and $\mathrm{ST} \geq 5 \mathrm{Y}$ groups. Changes in the effect of prognosis factors over time were determined by comparing the $\mathrm{ST}<5 \mathrm{Y}$ and $\mathrm{ST} \geq 5 \mathrm{Y}$ groups.

\section{Nomogram Construction and Validation}

Patients who survived for over 5 years were randomly divided into training and validation cohorts, with a split ratio of 7:3. Distinct parameters determined from univariate and multivariate Cox analyses were further output for the construction of nomogram models by R software 4.1.1 (R Development Core Team, R Foundation for Statistical Computing, Vienna, Austria). The nomograms were constructed to predict 10-year OS and CSS probability once patients survived for over 5 years. The concordance index (C-index) was used to compare the nomogram-predicted and observed outcomes. $\mathrm{C}$-index values between 0.7 and 1.0 indicate acceptable discriminatory capacity of the nomogram. The receiver operating characteristic curve (ROC) and the area under the ROC curve (AUC) were also calculated to evaluate the discriminative ability of the nomogram. Calibration curves were plotted to visually assess the agreement between the actual outcomes and the survival probabilities predicted by the nomograms. 


\section{Statistical Analyses}

Clinicopathological characteristics were compared using Pearson's chi-squared and Fisher's exact probability tests. Univariate and multivariate Cox proportional hazards models were applied to obtain hazard ratios (HRs) with 95\% confidence intervals (CIs) for the association between clinicopathological variables and OS or CSS. Independent prognostic factors were defined as those variables with $\mathrm{P}<0.05$ by multivariate Cox regression. Survival curves were generated using the Kaplan-Meier method, with Log rank tests employed to assess significance between subgroups. All statistical analyses were performed using SPSS Statistics 25.0 (IBM Corporation, Armonk, NY, USA). All statistical tests were two-sided, and a threshold of $\mathrm{P}<0.05$ was considered to be significant.

\section{Results}

\section{Comparison of Clinicopathological Characteristics and Treatment Strategies Between MA and SRCC Patients with ST $<5 Y$ and $S T \geq 5 Y$}

A total of 18,120 patients with MA and 2711 patients with SRCC were finally included in our study after screening. Among these, $8286 \mathrm{MA}$ patients (45.73\%) and 551 SRCC patients $(20.32 \%)$ had survived for over 5 years. Compared with MA and SRCC patients with ST $<5 \mathrm{Y}$, those who survived for over 5 years had higher frequency of right colon tumor, smaller tumor size, well differentiated tumor, lower AJCC stage; they also had lower incidence of tumor deposits, perineural invasion, and liver or lung metastasis, as well as lower CEA levels (all $\mathrm{P}<0.001$; Table 1 ). We also noted that patients with $\mathrm{ST} \geq 5 \mathrm{Y}$ tended to be younger, female, and married. However, there was no significant difference in age of diagnosis between $\mathrm{SRCC}$ patients with $\mathrm{ST}<5 \mathrm{Y}$ and those with $\mathrm{ST} \geq 5 \mathrm{Y}(\mathrm{P}=0.121)$. Concerning treatment strategies, relative to the $\mathrm{ST}<5 \mathrm{Y}$ group, a higher number of patients who survived for over 5 years received surgery $(\mathrm{P}<0.001)$, regional lymph node dissection $(\mathrm{P}<0.001$ ), and preoperative radiotherapy (MA: $\mathrm{P}<0.001$, SRCC: $\mathrm{P}=0.030$ ). In turn, patients with $\mathrm{ST}<5 \mathrm{Y}$ were more likely to have received chemotherapy $(\mathrm{P} \leq 0.001)$, whereas SRCC patients with $\mathrm{ST} \geq 5 \mathrm{Y}$ were more likely to have received systemic therapy $(\mathrm{P}<0.001)$.

\section{Prognostic Factors in MA and SRCC Patients with ST $\geq 5 Y$}

We next explored potential prognostic factors in patients with MA or SRCC who had survived for over 5 years. The clinical characteristics and treatment strategies significantly associated with OS and CSS were identified by univariate Cox regression analysis (Table 2). Meanwhile, results of multivariable Cox regression analysis (Table 3) revealed independent associations with worse overall survival of MA (MAOS) and MA cancer cause-specific survival (MACSS) for older age at diagnosis $(\mathrm{P}<0.001)$, rectum tumor $(\mathrm{P}=0.005, \mathrm{P}=0.014)$, advanced $\mathrm{T}$ stage (T3, $\mathrm{P}=0.008$, $\mathrm{P}=0.013$; T4, $\mathrm{P}=0.001, \mathrm{P}<0.001), \mathrm{N}$ stage $(\mathrm{P}<0.001)$, metastasis $(\mathrm{P}<0.001)$, and elevated CEA level $(\mathrm{P}<0.001)$. On the contrary, only N2 stage was identified as an independent prognostic factor for worse overall survival of SRCC (SRCCOS; $\mathrm{P}=0.024$ ) and SRCC cause-specific survival (SRCCSS; $\mathrm{P}=0.011$ ). Moreover, among patients diagnosed after 2010, tumor deposit was an independent prognostic indicator of MACSS in those with ST $\geq 5 \mathrm{Y}$ (Supplementary Table 1).

The receipt of surgery was independently associated with $49.1 \%$ decreased risk of overall mortality (HR, $0.509 ; 95 \%$ $\mathrm{CI}, 0.315-0.822 ; \mathrm{P}=0.006$ ) and $54.8 \%$ decreased risk of cancer-specific mortality (HR, $0.452 ; 95 \% \mathrm{CI}, 0.232-0.881$; $\mathrm{P}=0.020)$ in MA patients with $\mathrm{ST} \geq 5 \mathrm{Y}$. Chemotherapy exerted a significant positive effect (HR=0.801; 95\% CI, 0.6890.931; $\mathrm{P}=0.004$ ) on MAOS, but not on MACSS ( $\mathrm{HR}=1.151 ; 95 \% \mathrm{CI}, 0.893-1.482 ; \mathrm{P}=0.278$ ). However, the different treatment strategies offered little improvement on SRCCOS and SRCCSS, indicating that the SRCC histology is more likely to be associated with adverse prognosis.

\section{Differences in Prognostic Factors Between Patients with ST $<5 Y$ and ST $\geq 5 Y$}

Multivariable Cox regression analysis demonstrated that advanced $\mathrm{N}$ stage and metastasis were independently associated with inferior OS and CSS in both MA and SRCC patients with either ST $<5 \mathrm{Y}$ or ST $\geq 5 \mathrm{Y}$ (Table 4 and Supplementary Table 2). The independent prognostic factors for MAOS and MACSS in the ST $\geq 5 \mathrm{Y}$ group, including age, CEA level, surgery, and regional lymph nodes dissection, also had strong prognostic value in the ST $<5 \mathrm{Y}$ group. Kaplan-Meier curves showed that 
Table I Comparation of Clinical Characteristics of Patients with Different Survival Times

\begin{tabular}{|c|c|c|c|c|c|c|}
\hline \multirow[t]{2}{*}{ Characteristics } & \multicolumn{3}{|c|}{ MA } & \multicolumn{3}{|c|}{ SRCC } \\
\hline & $\begin{array}{l}S T<5 Y \\
(n=9834)\end{array}$ & $\begin{array}{l}S T \geq 5 Y \\
(n=8286)\end{array}$ & $P$ value & $\begin{array}{l}S T<5 Y \\
(n=2 \mid 60)\end{array}$ & $\begin{array}{l}S T \geq 5 Y \\
(n=55 I)\end{array}$ & $P$ value \\
\hline Age & & & $<0.001$ & & & 0.121 \\
\hline$\leq 49$ years & 1126 (1I.5) & II 177 (14.2) & & $483(22.4)$ & $108(19.6)$ & \\
\hline 50-64 years & $2458(25.0)$ & 2699 (32.6) & & $620(28.7)$ & $165(29.9)$ & \\
\hline $65-79$ years & 3377 (34.3) & $3062(37.0)$ & & $640(29.6)$ & $186(33.8)$ & \\
\hline$\geq 80$ years & $2873(29.2)$ & $1348(16.3)$ & & $417(19.3)$ & $92(16.7)$ & \\
\hline Sex & & & 0.003 & & & 0.026 \\
\hline Male & $4934(50.2)$ & $397 \mid$ (47.9) & & $1200(55.6)$ & $277(50.3)$ & \\
\hline Female & $4900(49.8)$ & $4315(52.1)$ & & $960(44.4)$ & $274(49.7)$ & \\
\hline Race & & & 0.006 & & & 0.013 \\
\hline White & 7939 (80.7) & $6762(81.6)$ & & $1727(80.0)$ & $468(84.9)$ & \\
\hline Black & 1248 (12.7) & $932(11.2)$ & & 239 (II.I) & $39(7.1)$ & \\
\hline Others & $647(6.6)$ & $592(7.1)$ & & $194(9.0)$ & $44(8.0)$ & \\
\hline Marital status & & & $<0.001$ & & & 0.102 \\
\hline Unmarried & 4810 (48.9) & 3265 (39.4) & & $958(44.4)$ & $219(39.7)$ & \\
\hline Married & 4637 (47.2) & $4719(57.0)$ & & 1117 (5I.7) & $313(56.8)$ & \\
\hline Unknown & 387 (3.9) & $302(3.6)$ & & $85(3.9)$ & $19(3.4)$ & \\
\hline Tumor location & & & $<0.001$ & & & 0.011 \\
\hline Right colon & $5628(57.2)$ & $5138(62.0)$ & & II 48 (53.I) & $332(60.3)$ & \\
\hline Left colon & $2816(28.6)$ & $2070(25.0)$ & & $573(26.5)$ & $125(22.7)$ & \\
\hline Rectum & $1390(14.1)$ & $1078(13.0)$ & & $439(20.3)$ & $94(17.1)$ & \\
\hline Tumor size & & & $<0.001$ & & & $<0.001$ \\
\hline$<50 \mathrm{~mm}$ & $3046(31.0)$ & $3516(42.4)$ & & $55 \mathrm{I}(25.5)$ & $229(4 I .6)$ & \\
\hline$\geq 50 \mathrm{~mm}$ & $543 \mid(55.2)$ & $4216(50.9)$ & & $1047(48.5)$ & $27 \mid(49.2)$ & \\
\hline Unknown & I357 (13.8) & $554(6.7)$ & & $562(26.0)$ & $5 \mathrm{I}(9.3)$ & \\
\hline Grade* & & & $<0.001$ & & & $<0.001$ \\
\hline I/II & $5972(60.7)$ & $6272(75.7)$ & & $97(4.5)$ & $45(8.2)$ & \\
\hline III/IV & $2506(25.5)$ & $1428(17.2)$ & & $1697(78.6)$ & 457 (82.9) & \\
\hline Unknown & 1356 (13.8) & $586(7.1)$ & & $366(16.9)$ & $49(8.9)$ & \\
\hline Stage & & & $<0.001$ & & & $<0.001$ \\
\hline 0/I & $579(5.9)$ & 1427 (I7.2) & & $48(2.2)$ & $67(12.2)$ & \\
\hline II & $2060(20.9)$ & $3627(43.8)$ & & 154 (7.I) & $167(30.3)$ & \\
\hline III & 3078 (31.3) & 2807 (33.9) & & 801 (37.I) & $278(50.5)$ & \\
\hline IV & $3855(39.2)$ & $330(4.0)$ & & $1069(49.5)$ & $24(4.4)$ & \\
\hline Unknown & $262(2.7)$ & $95(1.1)$ & & $88(4.1)$ & $15(2.7)$ & \\
\hline T stage & & & $<0.001$ & & & $<0.001$ \\
\hline T0/Tis/TI & $373(3.8)$ & $545(6.6)$ & & $107(5.0)$ & $48(8.7)$ & \\
\hline $\mathrm{T} 2$ & $568(5.8)$ & $1238(14.9)$ & & $31(1.4)$ & $48(8.7)$ & \\
\hline $\mathrm{T} 3$ & $48 I 1$ (48.9) & $5317(64.2)$ & & $745(34.5)$ & $348(63.2)$ & \\
\hline $\mathrm{T} 4$ & 3335 (33.9) & 1124 (13.6) & & $978(45.3)$ & 97 (17.6) & \\
\hline Unknown & $747(7.6)$ & $62(0.7)$ & & $299(13.8)$ & $10(1.8)$ & \\
\hline N stage & & & $<0.001$ & & & $<0.001$ \\
\hline No & 3497 (35.6) & $5201(62.8)$ & & $439(20.3)$ & $242(43.9)$ & \\
\hline $\mathrm{NI}$ & 2725 (27.7) & $2045(24.7)$ & & $450(20.8)$ & $135(24.5)$ & \\
\hline N2 & $3100(31.5)$ & $1002(12.1)$ & & $1059(49.0)$ & $166(30.1)$ & \\
\hline Unknown & $512(5.2)$ & $38(0.5)$ & & $212(9.8)$ & $8(1.5)$ & \\
\hline Metastasis & & & $<0.001$ & & & $<0.001$ \\
\hline Mo & $5843(59.4)$ & 7899 (95.3) & & $1063(49.2)$ & $517(93.8)$ & \\
\hline MI & 3855 (39.2) & $330(4.0)$ & & $1069(49.5)$ & $24(4.4)$ & \\
\hline
\end{tabular}

(Continued) 
Table I (Continued).

\begin{tabular}{|c|c|c|c|c|c|c|}
\hline \multirow[t]{2}{*}{ Characteristics } & \multicolumn{3}{|c|}{ MA } & \multicolumn{3}{|c|}{ SRCC } \\
\hline & $\begin{array}{l}S T<5 Y \\
(n=9834)\end{array}$ & $\begin{array}{l}S T \geq 5 Y \\
(n=8286)\end{array}$ & $P$ value & $\begin{array}{l}S T<5 Y \\
(n=2160)\end{array}$ & $\begin{array}{l}S T \geq 5 Y \\
(n=55 I)\end{array}$ & $P$ value \\
\hline Unknown & $136(1.4)$ & $57(0.7)$ & & $28(1.3)$ & $10(1.8)$ & \\
\hline Pretreatment CEA level & & & $<0.001$ & & & $<0.001$ \\
\hline Negative/Borderline & $2030(20.6)$ & $2940(95.3)$ & & $542(25.1)$ & $180(32.7)$ & \\
\hline Positive & $3990(40.6)$ & $1954(23.6)$ & & $926(42.9)$ & $144(26.1)$ & \\
\hline Unknown & $3814(38.8)$ & 3392 (40.9) & & $692(32.0)$ & $227(4 \mid .2)$ & \\
\hline Surgery of primary site & & & $<0.001$ & & & $<0.001$ \\
\hline No & $1377(14.0)$ & $70(0.8)$ & & $613(28.4)$ & II (2.0) & \\
\hline Yes & $8457(86.0)$ & $8216(99.2)$ & & I547 (7I.6) & $540(98.0)$ & \\
\hline $\begin{array}{l}\text { Regional lymph nodes } \\
\text { dissection }\end{array}$ & & & $<0.001$ & & & $<0.001$ \\
\hline None or biopsy & $1710(17.4)$ & $28 I(3.4)$ & & $68 I(31.5)$ & $30(5.4)$ & \\
\hline Yes & $8092(82.3)$ & $7993(96.5)$ & & $1460(67.6)$ & $520(94.4)$ & \\
\hline Unknown & $32(0.3)$ & $12(0.1)$ & & $19(0.9)$ & I (0.2) & \\
\hline Radiotherapy & & & $<0.001$ & & & 0.030 \\
\hline None & $8789(89.4)$ & $7230(87.3)$ & & $1917(88.8)$ & $465(84.4)$ & \\
\hline Preoperative RT & $554(5.6)$ & $668(8.1)$ & & $135(6.3)$ & $53(9.6)$ & \\
\hline Postoperative RT & $448(4.6)$ & $358(4.3)$ & & $99(4.6)$ & $30(5.4)$ & \\
\hline Others & $43(0.4)$ & $30(0.4)$ & & $9(0.4)$ & $3(0.5)$ & \\
\hline Chemotherapy & & & $<0.001$ & & & 0.001 \\
\hline No/Unknown & $5027(51.1)$ & $4829(58.3)$ & & $829(38.4)$ & $255(46.3)$ & \\
\hline Yes & 4807 (48.9) & 3457 (4I.7) & & $|33|(61.6)$ & $296(53.7)$ & \\
\hline Systemic therapy & & & $<0.001$ & & & $<0.001$ \\
\hline No & $4302(43.7)$ & $3404(41.1)$ & & $935(43.3)$ & $183(33.2)$ & \\
\hline Yes & $3019(30.7)$ & $2415(29.1)$ & & $769(35.6)$ & $215(39.0)$ & \\
\hline Unknown & $2513(25.6)$ & $2467(29.8)$ & & $456(21.1)$ & $153(27.8)$ & \\
\hline Tumor deposits** & & & $<0.001$ & & & $<0.001$ \\
\hline Negative & $2452(54.5)$ & $2408(85.3)$ & & $369(31.6)$ & $162(77.5)$ & \\
\hline Positive & $1053(23.4)$ & $204(7.2)$ & & $361(31.0)$ & $24(11.5)$ & \\
\hline Unknown & $991(22.0)$ & $212(7.5)$ & & $436(37.4)$ & $23(11.0)$ & \\
\hline Perineural invasion $* *$ & & & $<0.001$ & & & $<0.001$ \\
\hline No & 2875 (63.9) & $2378(84.2)$ & & $495(42.5)$ & $140(67.0)$ & \\
\hline Yes & $609(13.5)$ & I $44(5.1)$ & & $289(24.8)$ & $29(13.9)$ & \\
\hline Unknown & $1012(22.5)$ & $302(10.7)$ & & $382(32.8)$ & $40(19.1)$ & \\
\hline Liver metastasis** & & & $<0.001$ & & & $<0.001$ \\
\hline No & $3416(76.0)$ & $2752(97.5)$ & & $993(85.2)$ & $206(98.6)$ & \\
\hline Yes & $1003(22.3)$ & $57(2.0)$ & & $135(11.6)$ & $0(0.0)$ & \\
\hline Unknown & $77(1.7)$ & $15(0.5)$ & & $38(3.3)$ & $3(1.4)$ & \\
\hline Lung metastasis** & & & $<0.001$ & & & 0.001 \\
\hline No & 4077 (90.7) & 2800 (99.2) & & $1072(91.9)$ & $207(99.0)$ & \\
\hline Yes & $313(7.0)$ & $9(0.3)$ & & $54(4.6)$ & $0(0.0)$ & \\
\hline Unknown & $106(2.4)$ & $15(0.5)$ & & $40(3.4)$ & $2(1.0)$ & \\
\hline
\end{tabular}

Notes: *Grade I well differentiated, Grade II moderately differentiated, Grade III poorly differentiated, Grade IV undifferentiated. **Patients diagnosed between 20 I0 and 2018 were selected due to the detailed information of tumor deposit, perineural Invasion, liver and/or lung metastases were recorded since 2010.

Abbreviations: MA, mucinous adenocarcinoma; SRCC, signet ring cell carcinoma; ST, survival time; Y, year; RT, radiotherapy.

TNM stage was a stable and independent prognostic factor for CSS in both the $\mathrm{ST}<5 \mathrm{Y}$ and $\mathrm{ST} \geq 5 \mathrm{Y}$ groups (Figure $2 \mathrm{~A}$ and $\mathrm{B}$, Figure $3 \mathrm{~A}$ and $\mathrm{B}$ ). On the contrary, smaller tumor size and well differentiation, which were independently correlated with superior OS and CSS of MA (Figure 2C-F) and SRCC (Figure 3C-F) patients with ST $<5 \mathrm{Y}$, lost their predictive value in the 
Table 2 Prognostic Factors for the OS and CSS in Patients with a Survival Time Longer Than 5 Years by Univariate Analysis

\begin{tabular}{|c|c|c|c|c|c|c|c|c|}
\hline \multirow[t]{2}{*}{ Parameters } & \multicolumn{2}{|l|}{ MAOS } & \multicolumn{2}{|l|}{ MACSS } & \multicolumn{2}{|c|}{ SRCCOS } & \multicolumn{2}{|l|}{ SRCCSS } \\
\hline & HR (95\% Cl) & $P$ value & HR (95\% Cl) & $P$ value & HR (95\% Cl) & $P$ value & HR (95\% Cl) & $P$ value \\
\hline Age & I.067 (I.063, I.07I) & $<0.001$ & $1.008(1.002,1.013)$ & 0.005 & $1.047(1.034,1.060)$ & $<0.001$ & $0.994(0.978,1.010)$ & 0.455 \\
\hline \multicolumn{9}{|l|}{ Sex } \\
\hline Male & Reference & & Reference & & Reference & & Reference & \\
\hline Female & I.I73 (I.08I, I.274) & $<0.001$ & $0.952(0.824,1.100)$ & 0.504 & $1.462(1.060,2.016)$ & 0.021 & $1.222(0.739,2.023)$ & 0.435 \\
\hline \multicolumn{9}{|l|}{ Race } \\
\hline White & Reference & & Reference & & Reference & & Reference & \\
\hline Black & $0.865(0.756,0.989)$ & 0.034 & 1.339 (I.091, I.643) & 0.005 & I.I24 (0.590, 2.I4I) & 0.722 & $1.955(0.883,4.326)$ & 0.098 \\
\hline Others & $0.701(0.583,0.844)$ & $<0.001$ & $1.016(0.763,1.352)$ & 0.916 & $0.635(0.311,1.296)$ & 0.212 & $1.089(0.434,2.733)$ & 0.856 \\
\hline \multicolumn{9}{|l|}{ Marital status } \\
\hline Unmarried & Reference & & Reference & & Reference & & Reference & \\
\hline Married & $0.607(0.559,0.660)$ & $<0.001$ & $0.839(0.723,0.973)$ & 0.020 & $0.716(0.518,0.988)$ & 0.042 & $0.732(0.440,1.216)$ & 0.228 \\
\hline Unknown & $0.804(0.639,1.012)$ & 0.063 & $1.000(0.675,1.482)$ & 0.999 & $0.63 \mathrm{I}(0.230, \mathrm{I} .73 \mathrm{I})$ & 0.371 & $0.415(0.056,3.049)$ & 0.387 \\
\hline \multicolumn{9}{|c|}{ Tumor location } \\
\hline Right colon & Reference & & Reference & & Reference & & Reference & \\
\hline Left colon & $0.868(0.786,0.957)$ & 0.005 & $1.413(1.197,1.667)$ & $<0.001$ & $0.610(0.397,0.938)$ & 0.024 & $0.908(0.469,1.758)$ & 0.774 \\
\hline Rectum & $0.895(0.788,1.018)$ & 0.091 & $1.749(1.439,2.125)$ & $<0.001$ & $0.655(0.413,1.037)$ & 0.071 & $1.615(0.888,2.938)$ & 0.116 \\
\hline \multicolumn{9}{|l|}{ Tumor size } \\
\hline$<50 \mathrm{~mm}$ & Reference & & Reference & & Reference & & Reference & \\
\hline$\geq 50 \mathrm{~mm}$ & $0.991(0.910,1.079)$ & $0.84 I$ & $1.099(0.942,1.281)$ & 0.230 & $1.129(0.811,1.570)$ & 0.473 & $0.978(0.584,1.639)$ & 0.933 \\
\hline Unknown & $1.060(0.902,1.246)$ & 0.477 & $1.615(1.254,2.079)$ & $<0.001$ & $0.621(0.309,1.250)$ & 0.182 & $0.475(0.144,1.565)$ & 0.221 \\
\hline \multicolumn{9}{|l|}{ Grade* } \\
\hline I/II & Reference & & Reference & & Reference & & Reference & \\
\hline III/IV & $1.018(0.914,1.134)$ & 0.750 & $0.881(0.719,1.078)$ & 0.219 & I.279 (0.69I, 2.367) & 0.433 & $1.330(0.481,3.680)$ & 0.583 \\
\hline Unknown & $1.000(0.854,1.171)$ & 0.998 & $1.386(1.087,1.767)$ & 0.008 & $1.102(0.478,2.542)$ & 0.820 & $1.615(0.456,5.725)$ & 0.458 \\
\hline \multicolumn{9}{|l|}{ Stage } \\
\hline 0/l & Reference & & Reference & & Reference & & Reference & \\
\hline II & I.048 (0.932, I.I77) & 0.433 & $2.082(1.523,2.846)$ & $<0.001$ & $2.824(1.397,5.7 I I)$ & 0.004 & $2.375(0.526,10.718)$ & 0.260 \\
\hline III & $0.995(0.880,1.124)$ & 0.931 & $3.557(2.619,4.832)$ & $<0.001$ & $2.314(1.159,4.621)$ & 0.017 & $4.953(1.195,20.533)$ & 0.027 \\
\hline IV & $2.014(1.661,2.443)$ & $<0.001$ & I3.5।3 (9.639, 18.945) & $<0.001$ & $2.824(\mathrm{I} .05 \mathrm{I}, 7.584)$ & 0.039 & $10.532(2.125,52.205)$ & 0.004 \\
\hline Unknown & $1.528(1.090,2.140)$ & 0.014 & $5.327(3.020,9.395)$ & $<0.001$ & $3.836(1.364,10.789)$ & 0.011 & $10.22(1.87 \mid, 55.828)$ & 0.007 \\
\hline \multicolumn{9}{|l|}{ T stage } \\
\hline T0/Tis/TI & Reference & & Reference & & Reference & & Reference & \\
\hline $\mathrm{T} 2$ & $1.422(1.160,1.744)$ & 0.001 & $1.015(0.633,1.629)$ & 0.950 & $0.809(0.28 \mathrm{I}, 2.333)$ & 0.695 & $3.070(0.319,29.522)$ & 0.331 \\
\hline $\mathrm{T} 3$ & $1.357(1.129,1.631)$ & 0.001 & $2.125(1.421,3.176)$ & $<0.001$ & $2.173(1.058,4.462)$ & 0.034 & $5.463(0.749,39.86 \mathrm{I})$ & 0.094 \\
\hline
\end{tabular}


Table 2 (Continued).

\begin{tabular}{|c|c|c|c|c|c|c|c|c|}
\hline \multirow[t]{2}{*}{ Parameters } & \multicolumn{2}{|l|}{ MAOS } & \multicolumn{2}{|l|}{ MACSS } & \multicolumn{2}{|c|}{ SRCCOS } & \multicolumn{2}{|l|}{ SRCCSS } \\
\hline & HR (95\% Cl) & $P$ value & HR (95\% Cl) & $P$ value & HR (95\% Cl) & $P$ value & HR (95\% Cl) & $P$ value \\
\hline $\mathrm{T} 4$ & $1.454(1.180,1.793)$ & $<0.001$ & $3.499(2.295,5.336)$ & $<0.001$ & $2.303(1.048,5.058)$ & 0.038 & $10.849(1.447,81.325)$ & 0.020 \\
\hline Unknown & $2.564(1.690,3.889)$ & $<0.001$ & $7.436(4.015,13.77 I)$ & $<0.001$ & $6.299(2.046,19.397)$ & 0.001 & $21.44 \mid(2.223,206.816)$ & 0.008 \\
\hline \multicolumn{9}{|l|}{ N stage } \\
\hline No & Reference & & Reference & & Reference & & Reference & \\
\hline $\mathrm{NI}$ & $1.009(0.9 \mid 5,1.112)$ & 0.863 & $1.964(1.663,2.320)$ & $<0.001$ & 0.911 (0.60I, I.383) & 0.663 & $1.828(0.872,3.835)$ & 0.110 \\
\hline N2 & $1.047(0.920,1.191)$ & 0.486 & $2.817(2.335,3.397)$ & $<0.001$ & I.I78 (0.8I5, I.704) & 0.384 & $3.412(1.814,6.415)$ & $<0.001$ \\
\hline Unknown & $1.870(1.159,3.017)$ & 0.010 & $4.318(2.226,8.375)$ & $<0.001$ & $2.560(0.932,7.035)$ & 0.068 & $5.233(1.187,23.058)$ & 0.029 \\
\hline \multicolumn{9}{|l|}{ Metastasis } \\
\hline Mo & Reference & & Reference & & Reference & & Reference & \\
\hline MI & $1.967(1.658,2.334)$ & $<0.001$ & $5.553(4.562,6.759)$ & $<0.001$ & $1.226(0.574,2.619)$ & 0.599 & $2.919(1.253,6.800)$ & 0.013 \\
\hline Unknown & $1.224(0.796,1.881)$ & 0.357 & $1.200(0.537,2.681)$ & 0.657 & $1.410(0.522,3.809)$ & 0.499 & $3.043(0.950,9.748)$ & 0.061 \\
\hline \multicolumn{9}{|c|}{ Pretreatment CEA level } \\
\hline Negative/Borderline & Reference & & Reference & & Reference & & Reference & \\
\hline Positive & $1.420(1.275,1.581)$ & $<0.001$ & $2.088(1.735,2.512)$ & $<0.001$ & $1.469(0.975,2.213)$ & 0.066 & $1.225(0.643,2.335)$ & 0.538 \\
\hline Unknown & $1.145(1.039,1.261)$ & 0.006 & 1.221 (1.019, I.465) & 0.031 & $1.120(0.760,1.65 \mathrm{I})$ & 0.566 & $1.012(0.554,1.847)$ & 0.970 \\
\hline \multicolumn{9}{|c|}{ Surgery of primary site } \\
\hline No & Reference & & Reference & & Reference & & Reference & \\
\hline Yes & $0.459(0.324,0.65 \mathrm{I})$ & $<0.001$ & $0.222(0.145,0.340)$ & $<0.001$ & $\mathrm{I} .346(0.333,5.437)$ & 0.676 & $1.105(0.153,7.981)$ & 0.921 \\
\hline \multicolumn{9}{|c|}{ Regional lymph nodes dissection } \\
\hline None or biopsy & Reference & & Reference & & Reference & & Reference & \\
\hline Yes & $0.683(0.559,0.834)$ & $<0.001$ & $0.497(0.366,0.674)$ & $<0.001$ & $1.098(0.514,2.345)$ & 0.809 & $0.758(0.275,2.091)$ & 0.593 \\
\hline Unknown & I.I $44(0.466,2.808)$ & 0.769 & $2.551(1.012,6.435)$ & 0.047 & $7.848(0.959,64.255)$ & 0.055 & $9.498(1.054,85.616)$ & 0.045 \\
\hline \multicolumn{9}{|l|}{ Radiotherapy } \\
\hline None & Reference & & Reference & & Reference & & Reference & \\
\hline Preoperative RT & $0.766(0.646,0.908)$ & 0.002 & $1.598(1.275,2.003)$ & $<0.001$ & $1.119(0.665,1.881)$ & 0.672 & $2.443(1.294,4.614)$ & 0.006 \\
\hline Postoperative RT & $1.034(0.853,1.254)$ & 0.730 & $1.803(1.364,2.382)$ & $<0.001$ & $0.715(0.334,1.529)$ & 0.387 & $0.915(0.285,2.944)$ & 0.882 \\
\hline Others & $1.006(0.502,2.014)$ & 0.987 & $2.585(1.156,5.776)$ & 0.021 & & & & \\
\hline \multicolumn{9}{|l|}{ Chemotherapy } \\
\hline No/Unknown & Reference & & Reference & & Reference & & Reference & \\
\hline Yes & $0.664(0.609,0.725)$ & $<0.001$ & $1.890(1.633,2.187)$ & $<0.001$ & $0.677(0.492,0.932)$ & 0.017 & $2.066(1.192,3.583)$ & 0.010 \\
\hline \multicolumn{9}{|l|}{ Systemic therapy } \\
\hline No & Reference & & Reference & & Reference & & Reference & \\
\hline Yes & $0.687(0.611,0.771)$ & $<0.001$ & $1.792(1.486,2.161)$ & $<0.001$ & $0.739(0.488,1.118)$ & 0.152 & $1.779(0.940,3.367)$ & 0.077 \\
\hline Unknown & $0.909(0.827,1.000)$ & 0.051 & $1.407(1.168,1.694)$ & $<0.001$ & $0.847(0.569,1.259)$ & 0.412 & $1.08(0.524,2.223)$ & 0.835 \\
\hline
\end{tabular}




\begin{tabular}{|c|c|c|c|c|c|c|c|c|}
\hline \multicolumn{9}{|c|}{ Tumor deposits** } \\
\hline Negative & Reference & & Reference & & & & Reference & \\
\hline Unknown & $0.824(0.548,1.240)$ & 0.353 & $1.026(0.552,1.909)$ & 0.935 & $0.973(0.289,3.277)$ & 0.965 & $2.149(0.581,7.944)$ & 0.252 \\
\hline \multicolumn{9}{|c|}{ Perineural invasion** } \\
\hline No & Reference & & Reference & & & & Reference & \\
\hline Yes & $1.188(0.755,1.869)$ & 0.457 & $2.834(I .72 I, 4.666)$ & $<0.001$ & $0.876(0.310,2.7 \mid 5)$ & 0.876 & $1.552(0.4 \mathrm{II}, 5.862)$ & 0.517 \\
\hline Unknown & $1.108(0.812,1.513)$ & 0.517 & $1.232(0.756,2.006)$ & 0.402 & $0.552(0.162,1.874)$ & 0.340 & $1.252(0.332,4.722)$ & 0.740 \\
\hline \multicolumn{9}{|c|}{ Liver metastasis** } \\
\hline Unknown & I.70I $(0.546,5.302)$ & 0.360 & $4.347(1.384,13.656)$ & 0.012 & - & - & - & - \\
\hline \multicolumn{9}{|c|}{ Lung metastasis** } \\
\hline No & Reference & & Reference & & - & & - & \\
\hline Yes & $3.199(0.796,12.862)$ & 0.101 & $7.304(1.803,29.585)$ & 0.005 & - & - & - & - \\
\hline Unknown & $1.543(0.495,4.809)$ & 0.454 & $3.819(1.217,11.989)$ & 0.022 & - & - & - & - \\
\hline
\end{tabular}

Notes: *Grade I well differentiated, Grade II moderately differentiated, Grade III poorly differentiated, Grade IV undifferentiated. **Patients diagnosed between 2010 and 2018 were selected due to the detailed information of tumor deposit, perineural Invasion, liver and/or lung metastases were recorded since 2010.

Abbreviations: MAOS, overall survival of mucinous adenocarcinoma; MACSS, mucinous adenocarcinoma cancer cause-specific survival; SRCCOS, overall survival of signet ring cell carcinoma; SRCCSS, signet ring cell carcinoma cancer cause-specific survival; RT, radiotherapy; HR, hazard radio; Cl, confidence interval. 
Table 3 Multivariable Cox Regression Analysis of the Prognosis Factors Associated with OS and CSS in Patients with a Survival Time Longer Than 5 Years

\begin{tabular}{|c|c|c|c|c|c|c|c|c|}
\hline \multirow[t]{2}{*}{ Parameters } & \multicolumn{2}{|l|}{ MAOS } & \multicolumn{2}{|c|}{ MACSS } & \multicolumn{2}{|c|}{ SRCCOS } & \multicolumn{2}{|l|}{ SRCCSS } \\
\hline & HR $(95 \% \mathrm{Cl})$ & $P$ value & HR (95\% Cl) & $P$ value & HR (95\% Cl) & $P$ value & HR (95\% Cl) & $P$ value \\
\hline Age & $1.072(1.067,1.076)$ & $<0.001$ & $1.023(1.017,1.029)$ & $<0.001$ & $1.050(1.035,1.066)$ & $<0.001$ & $1.012(0.994,1.030)$ & 0.189 \\
\hline \multicolumn{9}{|l|}{ Sex } \\
\hline Male & Reference & & - & & Reference & & - & \\
\hline Female & $0.764(0.699,0.835)$ & $<0.001$ & - & - & $1.048(0.727,1.5 \mathrm{I} 2)$ & 0.801 & - & - \\
\hline \multicolumn{9}{|l|}{ Race } \\
\hline White & Reference & & Reference & & - & & - & \\
\hline Black & I.I25 (0.98I, I.290) & 0.093 & $1.442(1.169,1.778)$ & 0.001 & - & - & - & - \\
\hline Others & $0.803(0.667,0.967)$ & 0.021 & $0.991(0.743,1.32 I)$ & 0.949 & - & - & - & - \\
\hline \multicolumn{9}{|l|}{ Marital status } \\
\hline Unmarried & Reference & & Reference & & Reference & & - & \\
\hline Married & $0.752(0.688,0.822)$ & $<0.001$ & $0.904(0.776,1.054)$ & 0.198 & $0.963(0.678,1.368)$ & 0.835 & - & - \\
\hline Unknown & $0.956(0.758,1.205)$ & 0.702 & $1.073(0.720,1.599)$ & 0.729 & $0.815(0.288,2.302)$ & 0.699 & - & - \\
\hline \multicolumn{9}{|l|}{ Tumor location } \\
\hline Right colon & Reference & & Reference & & Reference & & - & \\
\hline Left colon & $1.058(0.957$, I.I7I) & 0.272 & $1.363(1.149,1.617)$ & $<0.001$ & $0.940(0.589,1.500)$ & 0.794 & - & - \\
\hline Rectum & $1.316(1.087,1.593)$ & 0.005 & $1.454(1.079,1.959)$ & 0.014 & $1.207(0.716,2.036)$ & 0.480 & - & - \\
\hline \multicolumn{9}{|l|}{ T stage } \\
\hline $\mathrm{TO} / \mathrm{Tis} / \mathrm{TI}$ & Reference & & Reference & & Reference & & Reference & \\
\hline $\mathrm{T} 2$ & I.274 (I.033, I.57I) & 0.024 & $1.041(0.644,1.682)$ & $0.87 I$ & $0.779(0.263,2.31 \mathrm{I})$ & 0.653 & $1.622(0.163,16.166)$ & 0.680 \\
\hline T3 & $1.303(1.073,1.583)$ & 0.008 & 1.706 (I.I2I, 2.597) & 0.013 & $1.735(0.805,3.742)$ & 0.160 & $2.765(0.36 \mathrm{I}, 21.170)$ & 0.327 \\
\hline $\mathrm{T} 4$ & $1.450(1.163,1.809)$ & 0.001 & $2.364(1.518,3.682)$ & $<0.001$ & $2.249(0.962,5.257)$ & 0.061 & $4.535(0.566,36.320)$ & 0.154 \\
\hline Unknown & $1.521(0.938,2.465)$ & 0.089 & $1.833(0.896,3.749)$ & 0.097 & $6.294(1.085,36.500)$ & 0.040 & & \\
\hline \multicolumn{9}{|l|}{ N stage } \\
\hline No & Reference & & Reference & & Reference & & Reference & \\
\hline $\mathrm{NI}$ & $1.209(0.141,1.345)$ & $<0.001$ & $1.719(1.432,2.064)$ & $<0.001$ & $1.054(0.668,1.662)$ & 0.822 & I.45। $(0.646,3.257)$ & 0.367 \\
\hline N2 & $1.391(9.141,1.603)$ & $<0.001$ & $2.249(1.823,2.773)$ & $<0.001$ & $1.630(1.068,2.487)$ & 0.024 & $2.589(1.249,5.368)$ & 0.011 \\
\hline Unknown & $0.800(0.449,1.423)$ & 0.447 & $0.688(0.306,1.548)$ & 0.366 & $0.929(0.136,6.331)$ & 0.940 & $0.281(0.023,3.408)$ & 0.319 \\
\hline \multicolumn{9}{|l|}{ Metastasis } \\
\hline Mo & Reference & & Reference & & Reference & & Reference & \\
\hline MI & $2.569(2.14 \mathrm{I}, 3.083)$ & $<0.001$ & $3.687(2.975,4.569)$ & $<0.001$ & $1.668(0.756,3.676)$ & 0.205 & $2.548(1.028,6.316)$ & 0.043 \\
\hline Unknown & I.I59 (0.73I, I.840) & 0.530 & $0.978(0.410,2.331)$ & 0.960 & $2.603(0.860,7.884)$ & 0.091 & $3.262(0.887,11.999)$ & 0.075 \\
\hline \multicolumn{9}{|c|}{ Pretreatment CEA level } \\
\hline Negative/Borderline & Reference & & Reference & & - & & - & \\
\hline Positive & $1.289(1.156,1.438)$ & $<0.001$ & $1.639(1.357,1.980)$ & $<0.001$ & - & - & - & - \\
\hline Unknown & $1.059(90.960,1.168)$ & 0.249 & I. $199(0.998,1.44 \mathrm{I})$ & 0.053 & - & - & - & - \\
\hline
\end{tabular}




\begin{tabular}{|c|c|c|c|c|c|c|c|c|}
\hline \multicolumn{9}{|c|}{ Surgery of primary site } \\
\hline No & Reference & & Reference & & - & & - & \\
\hline Yes & $0.509(0.315,0.822)$ & 0.006 & $0.452(0.232,0.88 I)$ & 0.020 & - & - & - & - \\
\hline \multicolumn{9}{|c|}{ Regional lymph nodes dissection } \\
\hline None or biopsy & Reference & & Reference & & - & & - & \\
\hline Yes & $0.791(0.614,1.019)$ & 0.070 & $0.582(0.380,0.890)$ & 0.013 & - & - & - & - \\
\hline Unknown & I.853 $(0.736,4.667)$ & 0.191 & $3.407(1.274,9.109)$ & 0.015 & - & - & - & - \\
\hline \multicolumn{9}{|l|}{ Radiotherapy } \\
\hline None & Reference & & Reference & & - & & Reference & \\
\hline Preoperative RT & $1.032(0.808,1.318)$ & 0.802 & $1.144(0.817,1.602)$ & 0.434 & - & - & $1.435(0.305,6.752)$ & 0.648 \\
\hline Postoperative RT & $1.330(1.061,1.667)$ & 0.013 & $1.216(0.882,1.675)$ & 0.232 & - & - & - & - \\
\hline Others & $2.195(1.079,4.467)$ & 0.030 & $2.613(1.128,6.053)$ & 0.025 & - & - & - & - \\
\hline \multicolumn{9}{|l|}{ Chemotherapy } \\
\hline No/Unknown & Reference & & Reference & & Reference & & Reference & \\
\hline Yes & $0.80 \mathrm{I}(0.689,0.93 \mathrm{I})$ & 0.004 & I.I5I (0.893, I.482) & 0.278 & $0.867(0.576,1.306)$ & 0.496 & $1.315(0.667,2.590)$ & 0.429 \\
\hline \multicolumn{9}{|l|}{ Systemic therapy } \\
\hline No & Reference & & Reference & & - & & - & \\
\hline Yes & $1.124(0.943,1.338)$ & 0.191 & $0.986(0.735,1.32 \mathrm{I})$ & 0.923 & - & - & - & - \\
\hline Unknown & $1.066(0.961,1.183)$ & 0.229 & $1.090(0.869,1.366)$ & 0.456 & - & - & - & - \\
\hline
\end{tabular}

Abbreviations: MAOS, overall survival of mucinous adenocarcinoma; MACSS, mucinous adenocarcinoma cancer cause-specific survival; SRCCOS, overall survival of signet ring cell carcinoma; SRCCSS, signet ring cell carcinoma cancer cause-specific survival; RT, radiotherapy; HR, hazard radio; Cl, confidence interval. 
Table 4 Multivariable Cox Regression Analysis of the Prognosis Factors Associated with OS and CSS in Patients with ST<5Y

\begin{tabular}{|c|c|c|c|c|c|c|c|c|}
\hline \multirow[t]{2}{*}{ Parameters } & \multicolumn{2}{|l|}{ MAOS } & \multicolumn{2}{|c|}{ MACSS } & \multicolumn{2}{|c|}{ SRCCOS } & \multicolumn{2}{|c|}{ SRCCSS } \\
\hline & HR (95\% Cl) & $P$ value & HR (95\% Cl) & P value & HR (95\% Cl) & $P$ value & HR (95\% Cl) & $P$ value \\
\hline Age & $1.008(1.006,1.009)$ & $<0.001$ & $1.005(1.003,1.007)$ & $<0.001$ & $1.009(1.006,1.012)$ & $<0.001$ & $1.007(1.004,1.010)$ & $<0.001$ \\
\hline \multicolumn{9}{|l|}{ Race } \\
\hline White & - & & Reference & & - & & - & \\
\hline Black & - & - & $1.059(0.990,1.132)$ & 0.075 & - & - & - & - \\
\hline Others & - & - & $1.089(0.998,1.188)$ & 0.038 & - & - & - & - \\
\hline \multicolumn{9}{|l|}{ Marital status } \\
\hline Unmarried & Reference & & - & & Reference & & - & \\
\hline Married & $0.943(0.905,0.983)$ & 0.005 & - & - & $0.90 \mathrm{I}(0.825,0.984)$ & 0.020 & - & - \\
\hline Unknown & $0.962(0.867,1.069)$ & 0.474 & - & - & $0.966(0.769,1.214)$ & 0.769 & - & - \\
\hline \multicolumn{9}{|c|}{ Tumor location } \\
\hline Right colon & Reference & & Reference & & - & & Reference & \\
\hline Left colon & $0.991(0.946,1.039)$ & 0.705 & $0.998(0.947,1.052)$ & 0.949 & - & - & $1.119(1.00 \mathrm{I}, 1.25 \mathrm{I})$ & 0.047 \\
\hline Rectum & $0.937(0.868,1.011)$ & 0.094 & $0.954(0.877,1.037)$ & 0.267 & - & - & $1.275(1.096,1.484)$ & 0.002 \\
\hline \multicolumn{9}{|l|}{ Tumor size } \\
\hline$<50 \mathrm{~mm}$ & Reference & & Reference & & Reference & & Reference & \\
\hline$\geq 50 \mathrm{~mm}$ & I.I3I (I.08I, I.I84) & $<0.001$ & $1.186(1.125,1.25 \mathrm{I})$ & $<0.001$ & $1.210(1.087,1.347)$ & 0.001 & 1.211 (1.080, I.359) & 0.001 \\
\hline Unknown & I.I43 (I.057, I.236) & 0.001 & $1.214(1.113,1.324)$ & $<0.001$ & $1.395(1.203,1.616)$ & $<0.001$ & $1.435(1.231,1.674)$ & $<0.001$ \\
\hline \multicolumn{9}{|l|}{ Grade* } \\
\hline $\mathrm{I} / \mathrm{II}$ & Reference & & Reference & & Reference & & Reference & \\
\hline III/IV & $1.168(1.113,1.225)$ & $<0.001$ & $1.193(1.131,1.259)$ & $<0.001$ & $1.270(1.028,1.568)$ & 0.026 & $1.339(1.065,1.683)$ & 0.012 \\
\hline Unknown & $1.099(1.026,1.176)$ & 0.007 & $1.123(1.042,1.210)$ & 0.002 & $1.394(1.104,1.759)$ & 0.005 & $1.470(1.145,1.888)$ & 0.003 \\
\hline \multicolumn{9}{|l|}{ T stage } \\
\hline T0/Tis/TI & Reference & & Reference & & Reference & & Reference & \\
\hline $\mathrm{T} 2$ & $1.048(0.913,1.203)$ & 0.507 & $0.789(0.655,0.950)$ & 0.012 & $0.559(0.370,0.845)$ & 0.006 & $0.449(0.274,0.736)$ & 0.001 \\
\hline T3 & $1.095(0.977,1.228)$ & 0.120 & $1.164(1.013,1.337)$ & 0.032 & $0.917(0.733,1.147)$ & 0.449 & $0.923(0.727,1.172)$ & 0.509 \\
\hline $\mathrm{T} 4$ & $1.307(1.164,1.468)$ & $<0.001$ & $1.524(1.326,1.75 \mathrm{I})$ & $<0.001$ & $1.019(0.819,1.269)$ & 0.865 & $\mathrm{I} .075(0.85 \mathrm{I}, \mathrm{I} .358)$ & 0.544 \\
\hline Unknown & I.198 (1.044, I.374) & 0.010 & I.34। (I.I47, I.567) & $<0.001$ & $0.982(0.779,1.238)$ & 0.877 & $1.032(0.806,1.321)$ & 0.803 \\
\hline \multicolumn{9}{|l|}{ N stage } \\
\hline No & Reference & & Reference & & Reference & & Reference & \\
\hline $\mathrm{NI}$ & $1.214(1.150,1.281)$ & $<0.001$ & $1.492(1.400,1.590)$ & $<0.001$ & $1.205(1.047,1.387)$ & 0.009 & 1.249 (I.07I, I.456) & 0.005 \\
\hline N2 & $1.528(1.443,1.619)$ & $<0.001$ & $1.977(1.850,2.113)$ & $<0.001$ & $1.637(1.422,1.884)$ & $<0.001$ & I.782 (I.527, 2.079) & $<0.001$ \\
\hline Unknown & $1.209(1.073,1.362)$ & 0.002 & 1.349 (I.191, I.529) & $<0.001$ & I.I $27(0.942$, I.349) & 0.193 & I.I6I (0.96I, I.403) & 0.122 \\
\hline \multicolumn{9}{|l|}{ Metastasis } \\
\hline Mo & Reference & & Reference & & Reference & & Reference & \\
\hline MI & $1.573(1.495,1.655)$ & $<0.001$ & $1.817(1.719,1.920)$ & $<0.001$ & $1.516(1.370,1.676)$ & $<0.001$ & $1.679(1.510,1.868)$ & $<0.001$ \\
\hline Unknown & $0.996(0.830,1.195)$ & 0.965 & $0.992(0.808,1.218)$ & 0.936 & I.I86 (0.795, I.769) & 0.403 & $1.222(0.789,1.891)$ & 0.370 \\
\hline
\end{tabular}




\begin{tabular}{|c|c|c|c|c|c|c|c|c|}
\hline \multicolumn{9}{|c|}{ Pretreatment CEA level } \\
\hline Negative/Borderline & Reference & & Reference & & Reference & & Reference & \\
\hline Positive & I.14I (1.080, I.206) & $<0.001$ & $1.222(1.147,1.302)$ & $<0.001$ & $1.215(1.087,1.359)$ & 0.001 & $1.215(1.082,1.365)$ & 0.001 \\
\hline Unknown & $1.044(0.989,1.103)$ & 0.120 & $1.090(1.022,1.162)$ & 0.009 & $1.064(0.947,1.195)$ & 0.298 & $1.052(0.930,1.189)$ & 0.418 \\
\hline \multicolumn{9}{|c|}{ Surgery of primary site } \\
\hline No & Reference & & Reference & & Reference & & Reference & \\
\hline Yes & $0.642(0.565,0.73 \mathrm{I})$ & $<0.001$ & $0.620(0.540,0.7 \mathrm{II})$ & $<0.001$ & $0.685(0.544,0.863)$ & 0.001 & $0.667(0.522,0.853)$ & 0.001 \\
\hline \multicolumn{9}{|c|}{ Regional lymph nodes dissection } \\
\hline None or biopsy & Reference & & Reference & & Reference & & Reference & \\
\hline Yes & $0.789(0.707,0.880)$ & $<0.001$ & $0.716(0.633,0.810)$ & $<0.001$ & $0.797(0.638,0.995)$ & 0.045 & $0.826(0.65 \mathrm{I}, 1.049)$ & 0.117 \\
\hline Unknown & $0.900(0.627,1.293)$ & 0.569 & $0.849(0.58 \mathrm{I}, \mathrm{I} .242)$ & 0.399 & $1.256(0.786,2.007)$ & 0.340 & $1.142(0.688,1.895)$ & 0.607 \\
\hline \multicolumn{9}{|l|}{ Radiotherapy } \\
\hline None & Reference & & Reference & & Reference & & Reference & \\
\hline Preoperative RT & $1.085(0.974,1.208)$ & 0.139 & I.I 78 (I.050, I.322) & 0.005 & $1.206(0.993,1.464)$ & 0.059 & $1.065(0.844,1.345)$ & 0.594 \\
\hline Postoperative RT & 0.985 (0.89I, I.090) & 0.773 & $1.055(0.948,1.173)$ & 0.326 & $1.000(0.812,1.233)$ & 0.998 & $0.945(0.756,1.180)$ & 0.616 \\
\hline Others & $0.975(0.719,1.323)$ & 0.873 & $0.995(0.723,1.372)$ & 0.978 & I.I $20(0.576,2.180)$ & 0.738 & I.II $(0.570,2.187)$ & 0.748 \\
\hline \multicolumn{9}{|l|}{ Chemotherapy } \\
\hline No/Unknown & Reference & & Reference & & Reference & & Reference & \\
\hline Yes & $0.71 ।(0.663,0.764)$ & $<0.001$ & $0.765(0.723,0.810)$ & $<0.001$ & $0.625(0.547,0.715)$ & $<0.001$ & $0.633(0.549,0.728)$ & $<0.001$ \\
\hline \multicolumn{9}{|l|}{ Systemic therapy } \\
\hline No & Reference & & - & & Reference & & Reference & \\
\hline Yes & $0.948(0.876,1.025)$ & 0.182 & - & - & $0.994(0.85 \mathrm{I}, \mathrm{I} .16 \mathrm{I})$ & 0.938 & $1.000(0.949,1.053)$ & 0.588 \\
\hline Unknown & $0.933(0.882,0.986)$ & 0.014 & - & - & $0.934(0.822,1.062)$ & 0.297 & $0.909(0.858,0.963)$ & 0.620 \\
\hline
\end{tabular}

Notes: *Grade I well differentiated, Grade II moderately differentiated, Grade III poorly differentiated, Grade IV undifferentiated.

Abbreviations: MAOS, overall survival of mucinous adenocarcinoma; MACSS, mucinous adenocarcinoma cancer cause-specific survival; SRCCOS, overall survival of signet ring cell carcinoma; SRCCSS, signet ring cell carcinoma cancer cause-specific survival; ST, survival time; RT, radiotherapy; HR, hazard radio; Cl, confidence interval. 


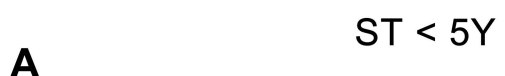

A

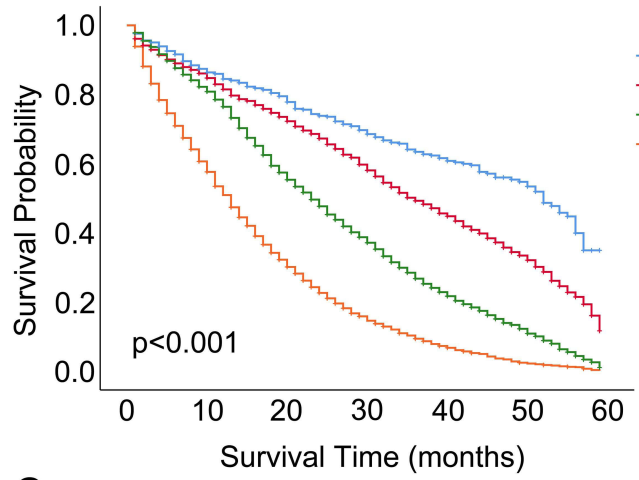

C

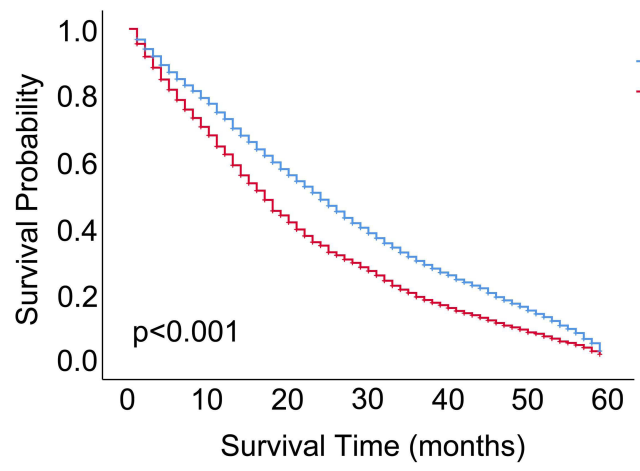

$\mathbf{E}$

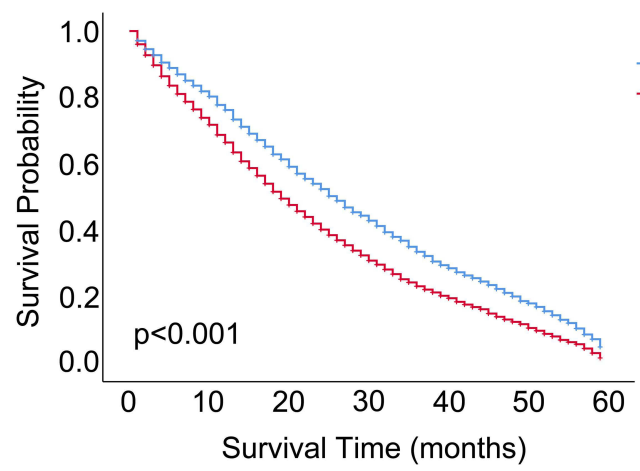

$\mathrm{ST} \geq 5 \mathrm{Y}$

B

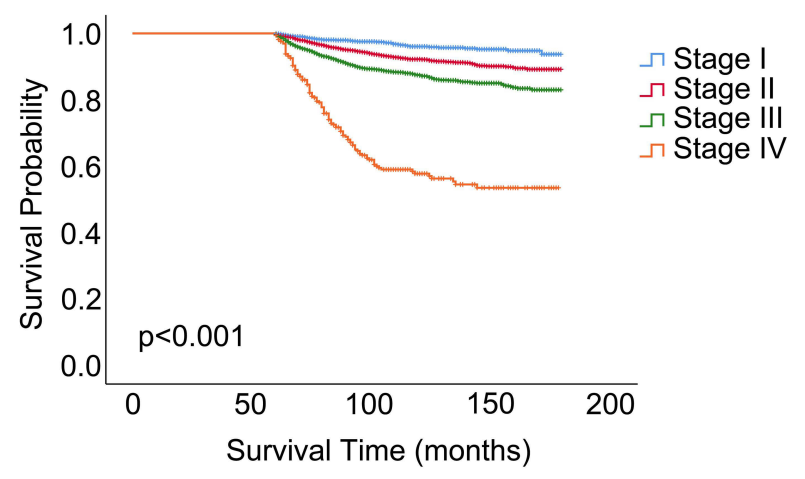

D

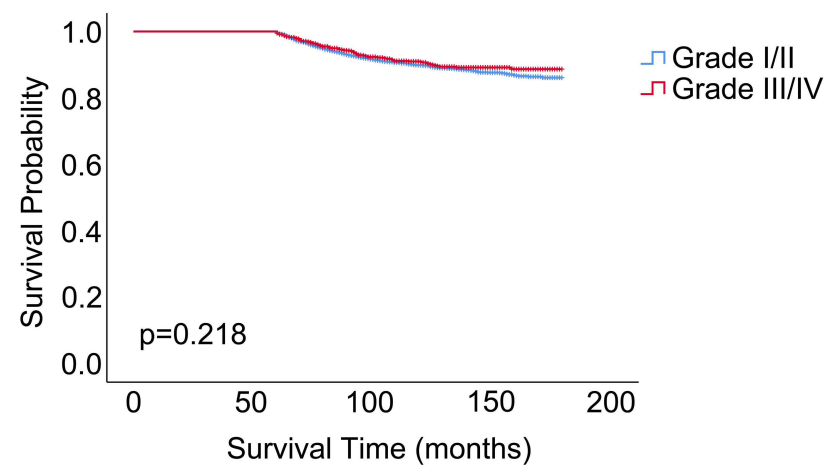

$\mathbf{F}$

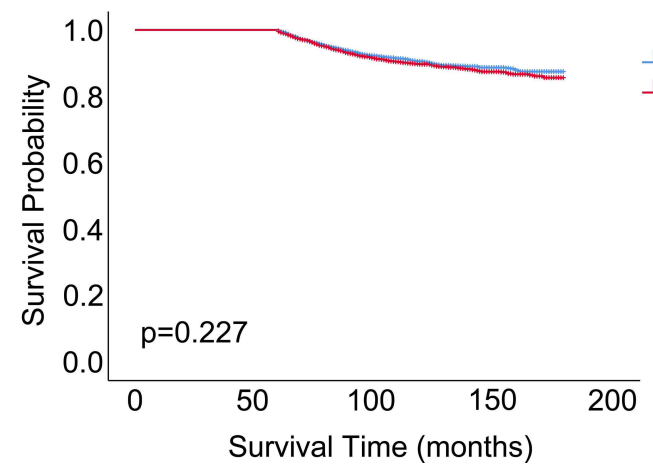

Figure 2 Kaplan-Meier curves for CSS in MA patients with $\mathrm{ST}<5 \mathrm{Y}$ and $\mathrm{ST} \geq 5 \mathrm{Y}$.

Notes: (A, C and E) MA patients with ST<5Y; (A) TNM stage; (C) tumor grade; (E) tumor size. (B, D and F) MA patients with ST $\geq 5 Y$; (B) TNM stage; (D) tumor grade; (F) tumor size.

Abbreviations: CSS, cancer-specific survival; MA, mucinous adenocarcinoma; ST, survival time; Y, year.

$\mathrm{ST} \geq 5 \mathrm{Y}$ group. Compared to MA patients with right colon tumor, a higher hazard of CCS mortality was noted in MA patients with left colon $(\mathrm{HR}=1.363 ; 95 \% \mathrm{CI}, 1.149-1.617 ; \mathrm{P} \leq 0.001)$ or rectum tumor $(\mathrm{HR}=1.454 ; 95 \% \mathrm{CI}, 1.079-1.959 ; \mathrm{P}=0.014)$ in the $\mathrm{ST} \geq 5 \mathrm{Y}$ group; however, neither left colon nor rectum tumor location conferred differential risk in the $\mathrm{ST}<5 \mathrm{Y}$ group $(\mathrm{P}=0.949, \mathrm{P}=0.267$, respectively). In addition, receipt of surgery of primary site was associated with $31.5 \%(\mathrm{P}=0.001)$ and $33.3 \%$ ( $\mathrm{P}=0.001)$ decreased risk of overall and cancer-specific mortality, respectively, in SRCC patients with ST $<5 \mathrm{Y}$. However, surgery was not associated with prognosis of SRCC patients with $\mathrm{ST} \geq 5 \mathrm{Y}$. 

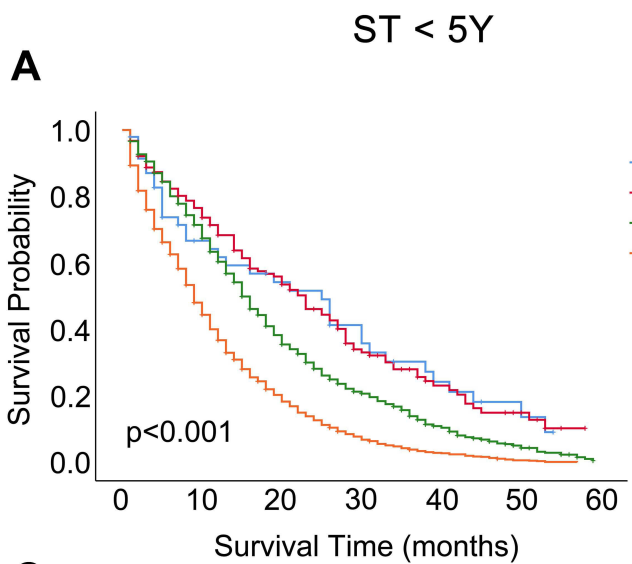

C
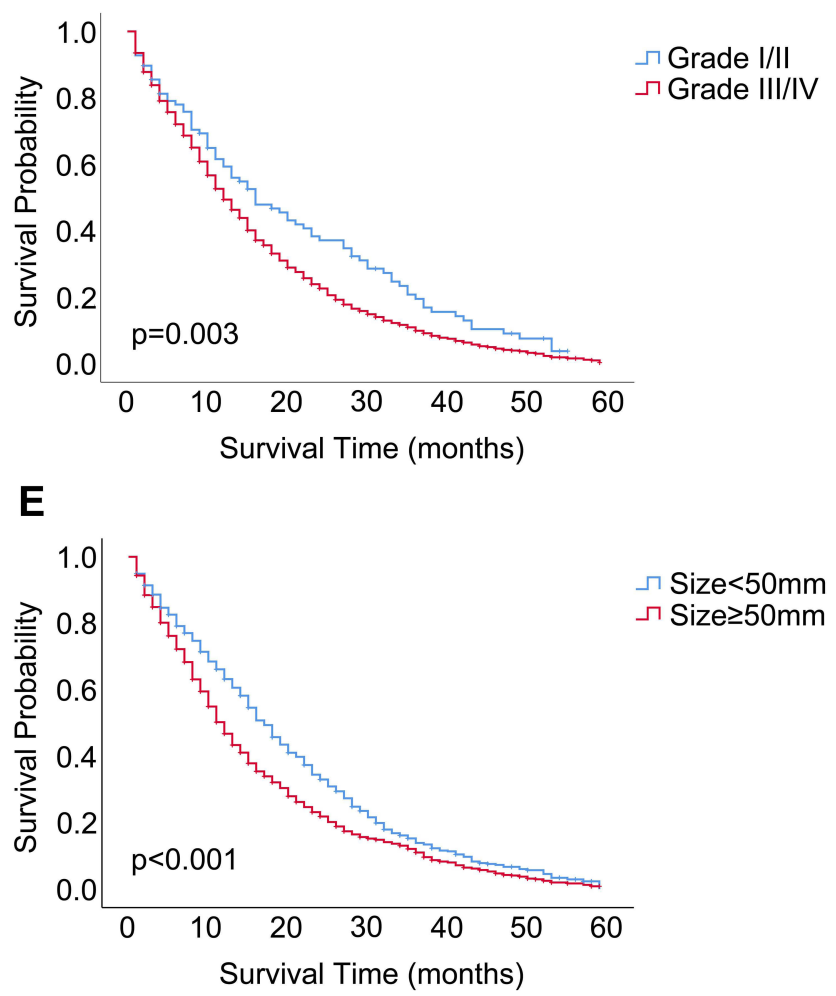

$\mathrm{ST} \geq 5 \mathrm{Y}$

B
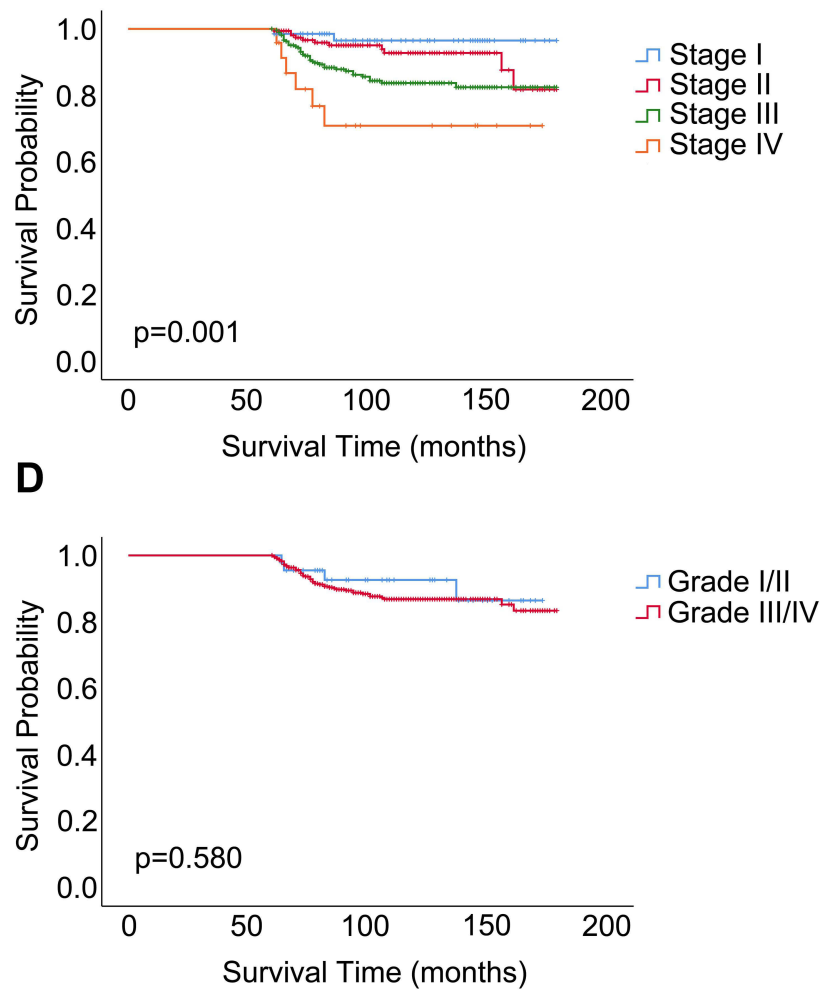

$\mathbf{F}$

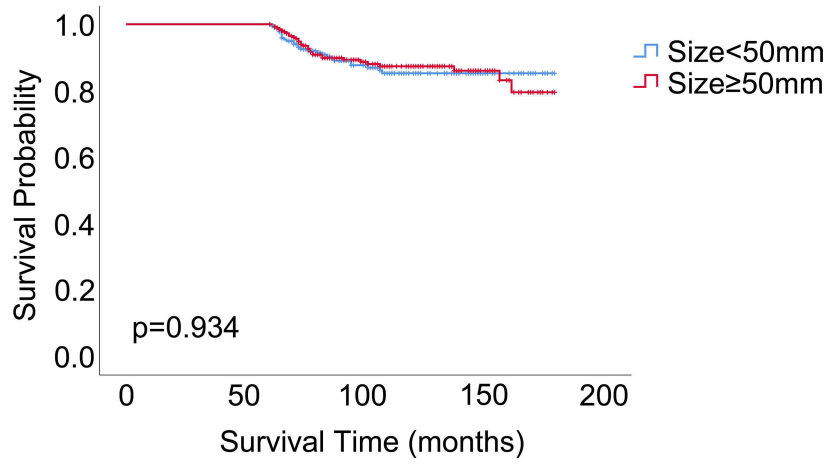

Figure 3 Kaplan-Meier curves for CSS in SRCC patients with ST $<5 Y$ and ST $\geq 5 Y$.

Notes: (A, C and E) MA patients with ST<5Y; (A) TNM stage; (C) tumor grade; (E) tumor size. (B, D and F) MA patients with ST $\geq 5$ Y; (B) TNM stage; (D) tumor grade; (F) tumor size.

Abbreviations: CSS, cancer-specific survival; SRCC, signet ring cell carcinoma; ST, survival time; Y, year.

\section{Weakened Survival Benefit of Chemotherapy in MA and SRCC Patients Surviving Over 5 Years}

To evaluate whether the survival benefit of chemotherapy changed over time, we compared the survival improvement offered by chemotherapy in patients with $\mathrm{ST}<5 \mathrm{Y}$ and $\mathrm{ST} \geq 5 \mathrm{Y}$ (Figure 4). The Kaplan-Meier curves indicated that receipt of chemotherapy was independently associated with better OS and CSS in MA and SRCC patients with ST $<5 \mathrm{Y}$ (all $\mathrm{P}<0.001$ ) (Figure 4A, C, E, and $\mathrm{G}$ ), whereas this benefit weakened or even disappeared in patients with $\mathrm{ST} \geq 5 \mathrm{Y}$ (Figure 4B, D, F, and H). Specifically, multivariate analysis showed that the receipt of chemotherapy significantly decreased the risk of overall mortality (by $19.9 \% ; \mathrm{P}=0.004$ ) in MA patients with $\mathrm{ST} \geq 5 \mathrm{Y}$. 
$\mathrm{ST}<5 \mathrm{Y}$

A

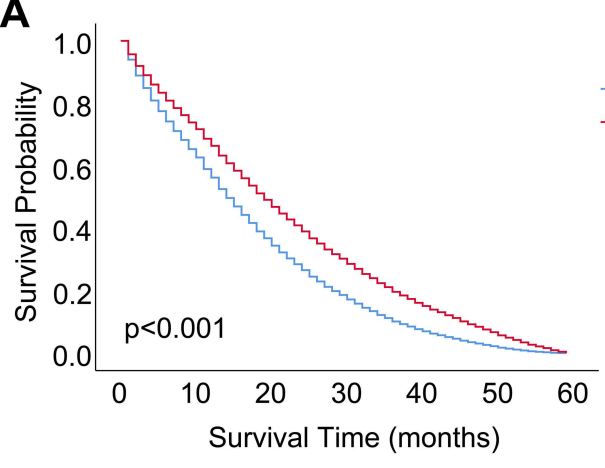

C

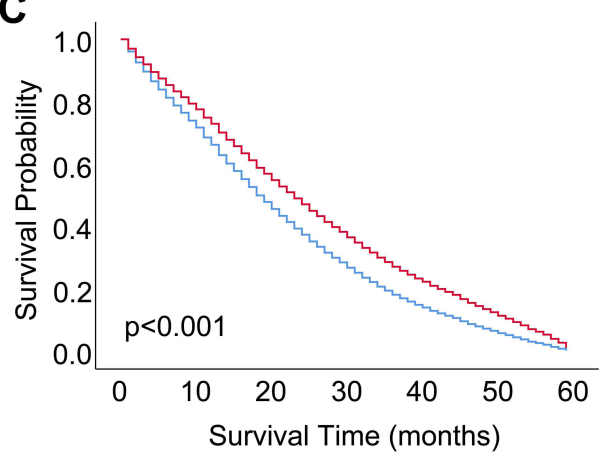

E

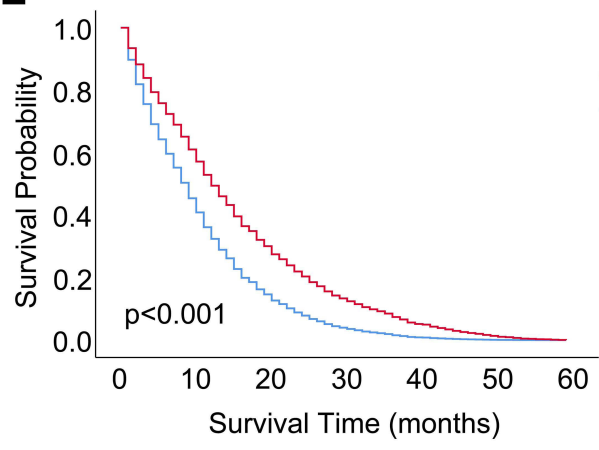

G

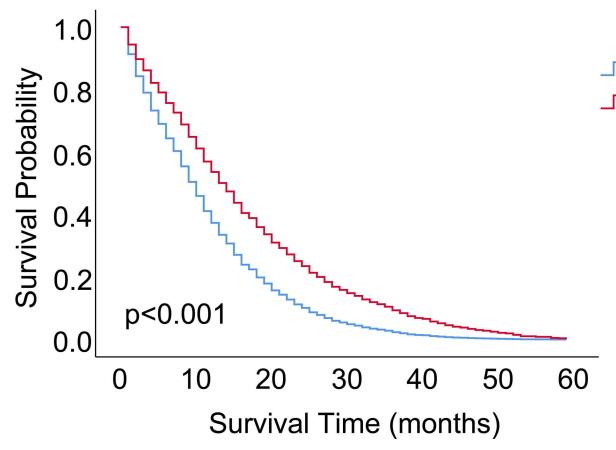

$\mathrm{ST} \geq 5 \mathrm{Y}$

B

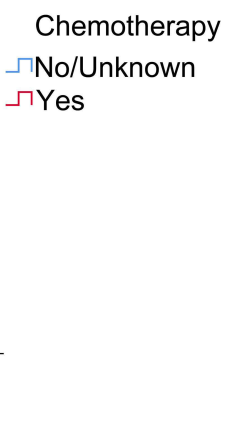

Chemotherapy

$\neg$ No/Unknown $\neg$ Yes

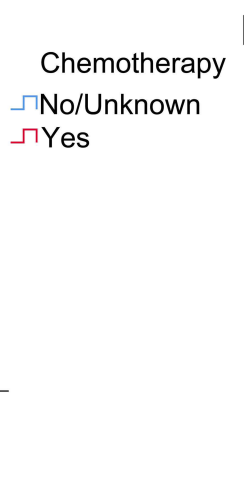

D

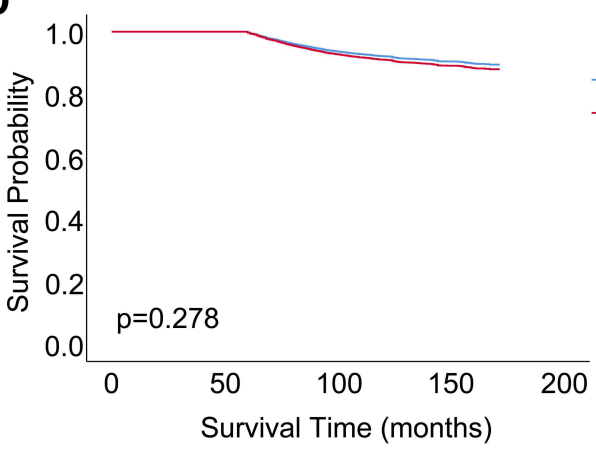

Chemotherapy $\neg \mathrm{No} /$ Unknown $\neg$ Yes

$\mathbf{F}$
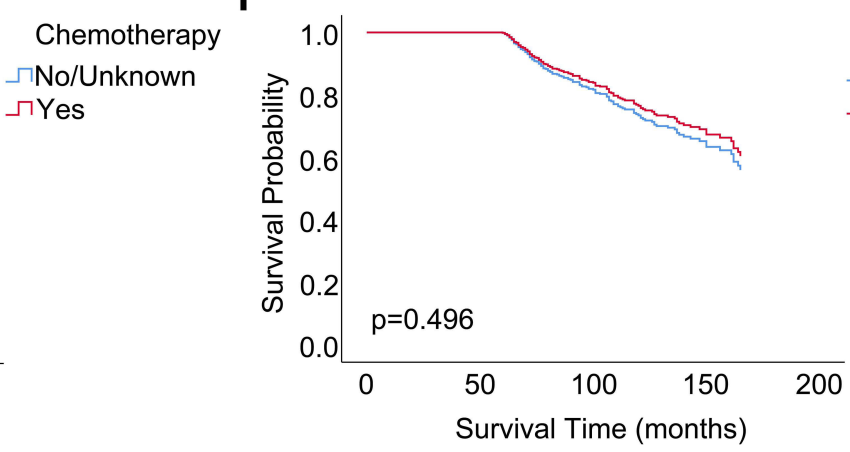

Chemotherapy $\neg \mathrm{No} /$ Unknown $\neg$ Yes

H

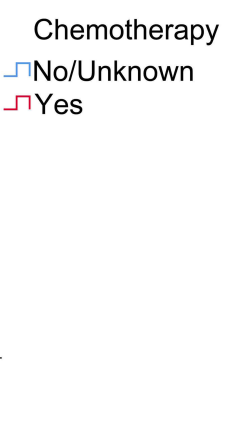

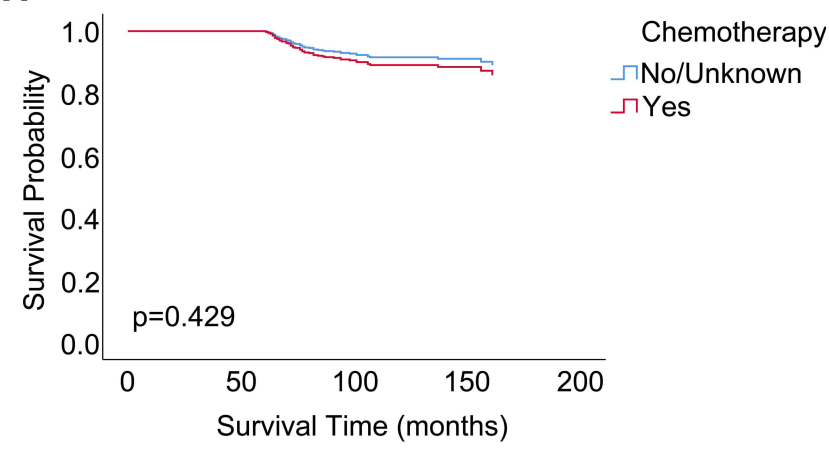

Figure 4 Survival analysis of chemotherapy in MA and SRCC patients with $S T<5 Y$ and $S T \geq 5 Y$.

Notes: (A, C, E and G) Patients with ST<5Y; (B, D, F and H) patients with ST $\geq 5$ Y; (A and B) overall survival of mucinous adenocarcinoma; (C and D) cancer causespecific survival of mucinous adenocarcinoma; (E and $\mathbf{F})$ overall survival of signet ring cell carcinoma; $(\mathbf{G}$ and $\mathbf{H})$ cause-specific survival of signet ring cell carcinoma cancer. 
We further explored the prognostic value of chemotherapy in MA patients with ST $\geq 5 \mathrm{Y}$ stratified by AJCC stage and tumor location (Figure 5). Kaplan-Meier curves revealed significantly better OS for chemotherapy in MA patients with right colon or left colon tumors (Figure 5B and C), but not in those with rectum tumor (Figure 5D). Moreover, stage IIIII MA patients who received chemotherapy had a significantly better OS (Figure 5F and G), while no significant impact of chemotherapy on OS was detected for stage I and stage IV patients in the two survival groups (Figure 5E and $\mathrm{H}$ ).

\section{Prognostic Nomograms for MA Patients Surviving Longer Than 5 Years}

Nomograms were constructed based on the significant prognostic factors identified by multivariate Cox regression. The final nomogram models were specifically developed to predict the 10-year OS and CSS probability of MA patients who survived for over 5 years (Figure 6). In the nomogram estimation system, a weighted point value was attributed to each factor that implied a contribution to the survival prognosis. By drawing a perpendicular line from the variable value to the axis labeled "Total Points" to calculate the scores of all variables, the 10-year survival probabilities corresponding to the total points could be obtained. The predictive accuracy of the final nomogram system was determined by calculating the Harrell's C-index. The C-index values of the nomogram predicting OS were 0.717 (95\% CI, 0.704-0.730) and 0.727 (95\% CI, 0.706-0.748) in the training and validation cohorts, respectively, indicating acceptable discrimination capacity. Similarly, good discrimination ability was observed for the nomogram predicting CSS, for which C-index values in the training and validation groups were 0.712 (95\% CI, 0.688-0.736) and 0.735 (95\% CI, 0.699-0.771), respectively.

\section{Calibration and Validation of Prognostic Nomograms}

We next validated the nomograms both internally, in the training cohort, and externally, in the validation cohort (Figure 7). In calibration plots, the diagonal reference line denotes equality between the actual survival and the predicted survival probability. Thus, the closeness of the solid line to the diagonal line in the calibration plots depicted in Figure 7B, D, F, and H reveals good agreement between actual observations and nomograms' predicted probabilities, ensuring the repeatability as well as the reliability of the constructed models. We further evaluated the effectiveness of the nomograms by plotting ROC curves. The corresponding AUC values of the nomograms for 10-year OS and CSS were 0.755 and 0.706 in the training cohort (Figure 7A and E) and 0.768 and 0.732 in the validation cohort (Figure 7C and G), respectively. An AUC value between 0.7 and 1.0 indicates good discriminatory capacity of the nomogram. These findings indicated that the nomograms constructed in this study were strong prognostic predictors for estimating longterm OS and CSS probability for MA patients who have survived for over 5 years.

\section{Discussion}

MA and SRCC are well defined histopathologic subtypes of CRC, characterized by clinical heterogeneity and distinct survival outcomes. ${ }^{6,7}$ Colorectal SRCC and MA were considered to be associated with poor prognosis in many previous studies. ${ }^{12-14}$ Considering that more and more patients with MA or SRCC are living longer lives, as well as the reported changes in the impact of prognostic factors for CSS over time, ${ }^{15,16}$ the need arises to identify reliable long-term prognostic factors for patients who survived for over 5 years to promote long-term CRC management, especially for early-onset patients. Thus, in this study we investigated the prognostic values of clinicopathological characteristics and treatment outcomes in MA and SRCC patients who survived for over 5 years. Furthermore, we developed novel prognostic nomograms to predict 10-year OS and CSS probability specifically for these patients, and calibration plots showed high quality and acceptable discrimination of the nomograms.

Our study demonstrated that various clinical characteristics of MA and SRCC patients who survived for over 5 years differed significantly from those of patients that survived less than 5 years. MA and SRCC patients with ST $\geq 5 \mathrm{Y}$ were more likely to be younger, female, married, diagnosed at early stage, have well differentiated and smaller tumors, and present lower frequencies of tumor deposit, perineural invasion, and elevated CEA. Moreover, we confirmed that the prognostic value of tumor size, tumor differentiation, surgery, and chemotherapy weakened over time and even disappeared after a long period of follow-up time. These results highlighted the necessity of dynamic prognosis 
A

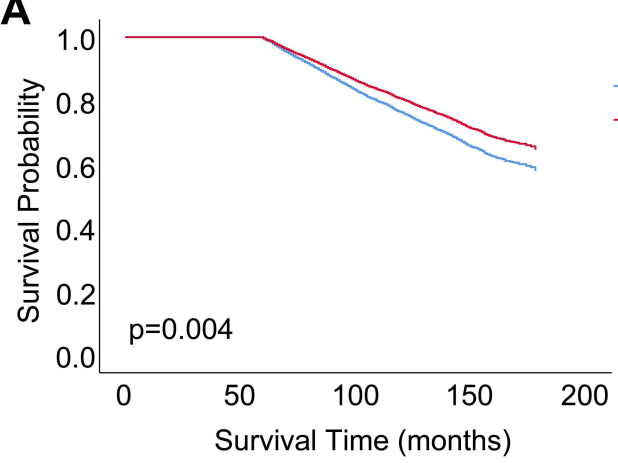

C

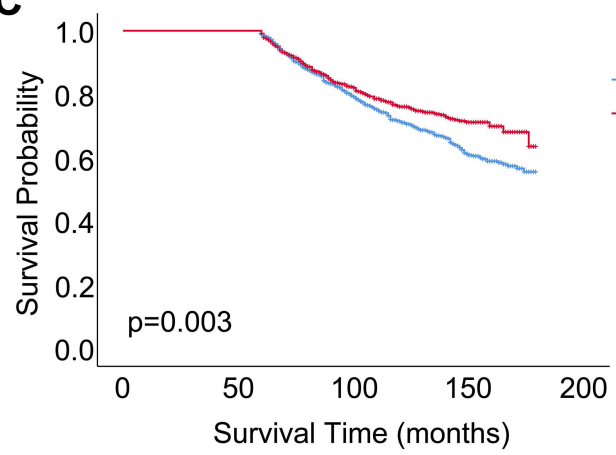

E

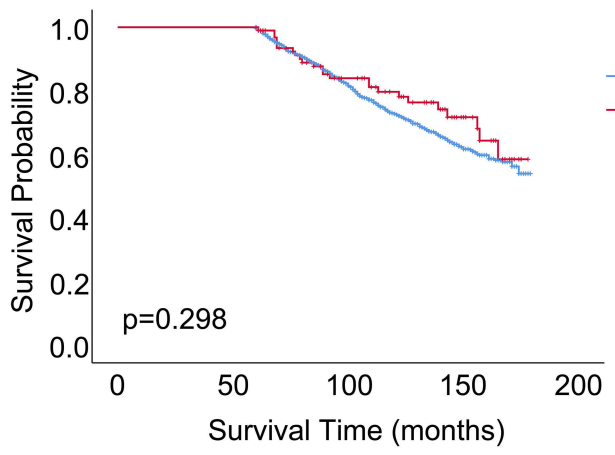

G

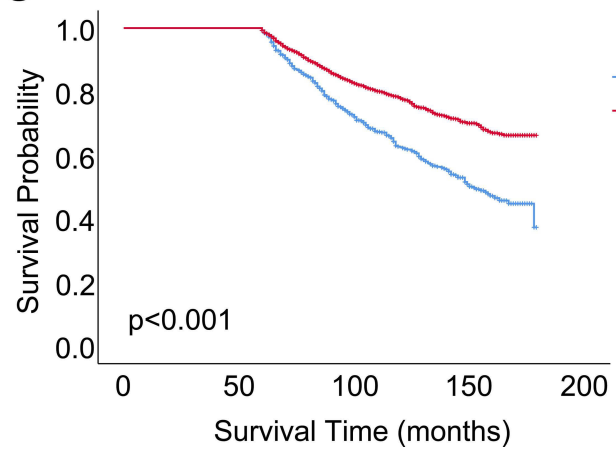

B

Chemotherapy $\neg$ No/Unknown $\neg$ Yes

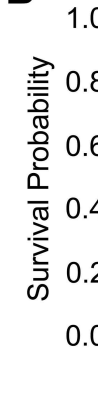

Right colon $\neg$ No/Unknown $\neg$ Yes

\section{D}
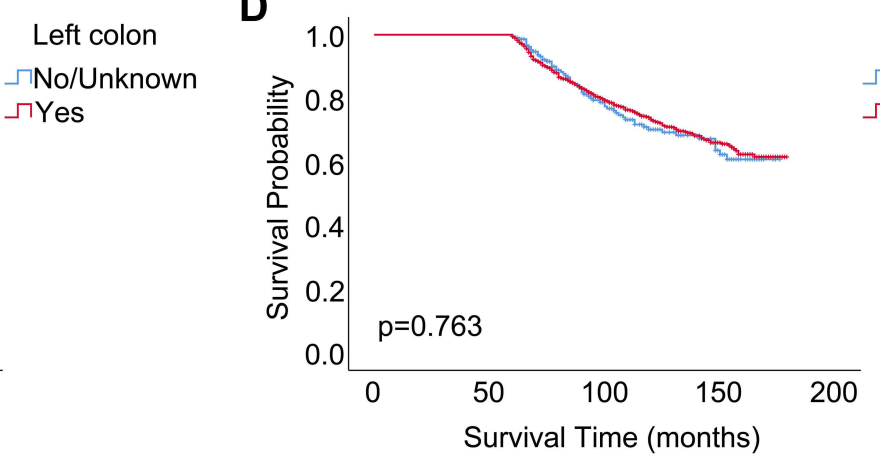

Rectum

$\neg$ No/Unknown $\neg$ Yes

F
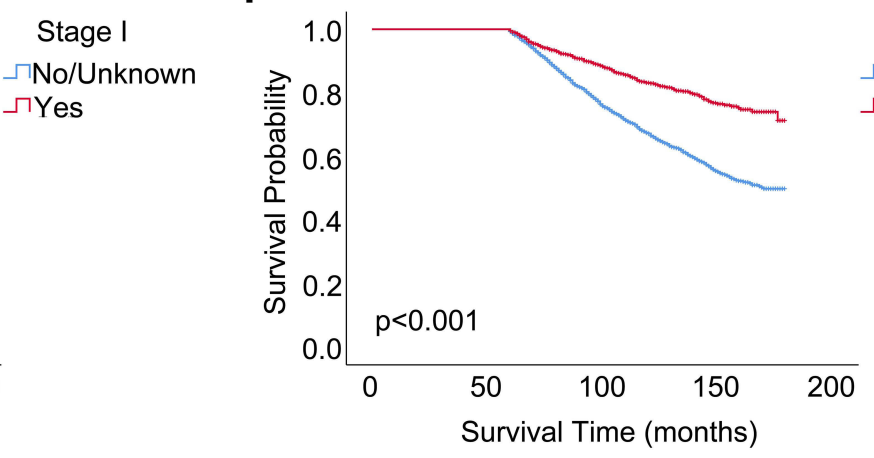

Stage II

$\neg$ No/Unknown $\neg$ Yes

H

Stage III

$\neg$ No/Unknown

$\neg$ Yes

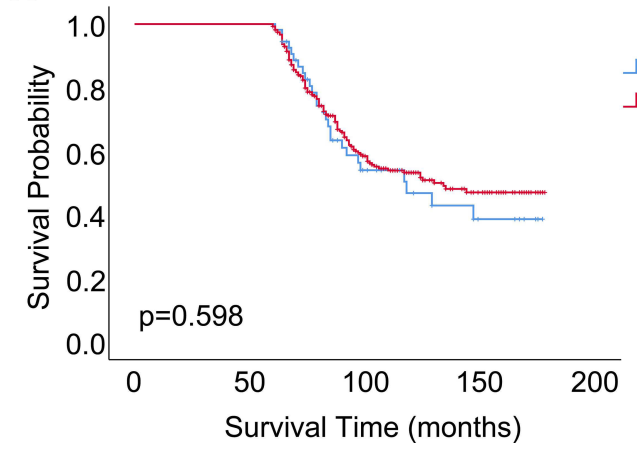

Stage IV

$\neg$ No/Unknown

$\neg$ Yes

Figure 5 Survival benefit of chemotherapy for MAOS in patients with $S T \geq 5 Y$.

Notes: (A) The whole cohort; (B) patients with primary tumor located in the right colon; (C) patients with primary tumor located in the left colon; (D) patients with primary tumor located in the rectum; (E) patients with stage I; (F) patients with stage II; (G) patients with stage III; $(\mathbf{H})$ patients with stage IV.

Abbreviations: MAOS, overall survival of mucinous adenocarcinoma; ST, survival time; Y, year. 
A

Points

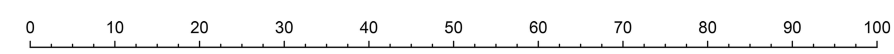

Age

\begin{tabular}{|c|c|}
\hline $50-64$ & $->-8$ \\
\hline
\end{tabular}

Sex

Race

Female

Female

Others $\quad$ Bhite

Marital status

Unknown

Married Unmarried

Left colon

Tumor location

Right colon Rectum

T stage

$\mathrm{N}$ stage

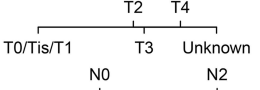

Metastasis

Unknown N1

Unknown

MO

Unknown

CEA level

Negative/Borderline Positive

Surgery

LN dissection

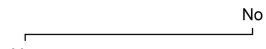

None or biopsy

Yes Unknown

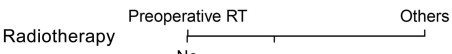

Chemotherapy

No/Unknown

Yes

Total Points

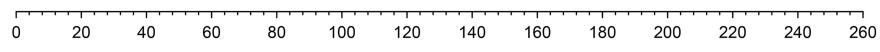

10-year OS Probability

\begin{tabular}{llllllllll}
\hline 0.9 & 0.8 & 0.7 & 0.6 & 0.5 & 0.4 & 0.3 & 0.2 & 0.1 & 0.05
\end{tabular}

B

Points

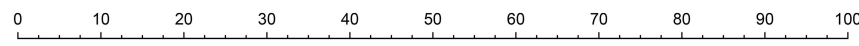

Age

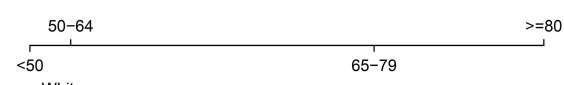

Race

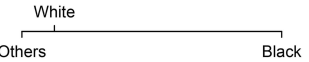

Marital status Married Unknown

Unmarried

Tumor location $\underset{\text { Right colon }}{\stackrel{\text { Left colon }}{\text { Rectum }}}$

T stage $\quad \stackrel{\mathrm{T} 2}{\mathrm{~T} / \mathrm{Tis} / \mathrm{T} 1} \stackrel{\mathrm{T}}{\mathrm{T} 3}^{\mathrm{T}}$

$\mathrm{N}$ stage

Unknown $\quad \stackrel{\text { No }}{n} \mathrm{~N}^{\mathrm{N} 1}$

Unknown

MO

nknown

Metastasis

CEA level

Negative/Borderline Positive

Surgery

LN dissection

$$
\text { Yes }
$$

Yes

Radiotherapy

Preoperative RT

No Postoperative RT Yes

Chemotherapy

No/Unknown

Total Points$$
\text { r. }
$$

10-year CSS Probability

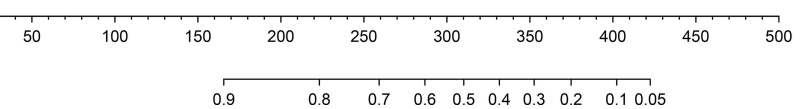
None or biopsy

Figure 6 Construction of nomograms to predict the 10-year OS and CSS probability for MA patients with ST $\geq 5$ Y.

Notes: (A) Nomogram to predict the OS probability; (B) nomogram to predict the CSS probability.

Abbreviations: MA, mucinous adenocarcinoma; OS, overall survival; CSS, cancer-specific survival; CEA, carcinoembryonic antigen; LN, lymph nodes; RT, radiotherapy; ST, survival time; Y, year. 
A

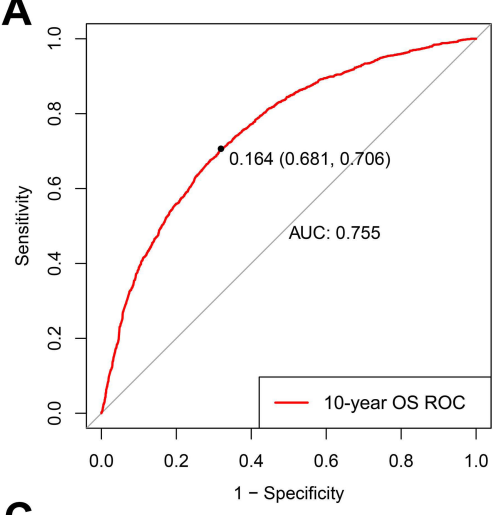

C

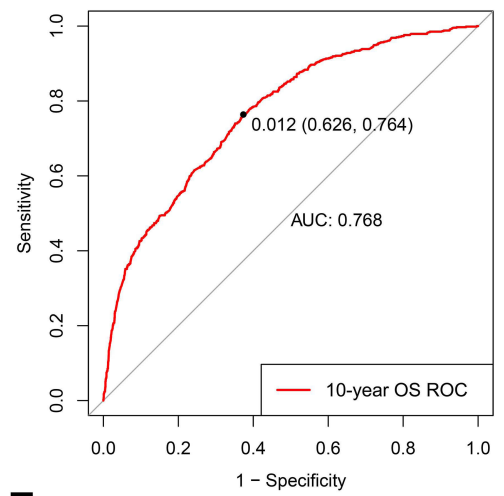

E

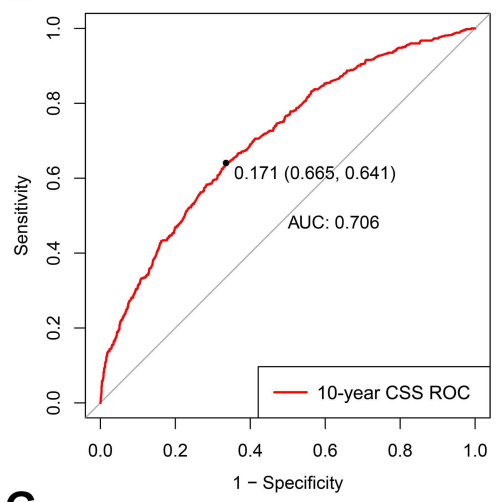

G

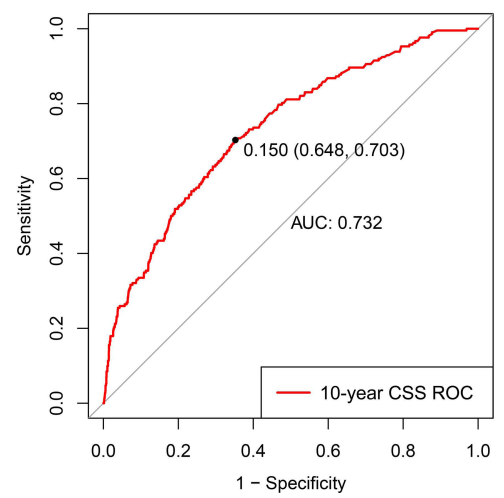

B

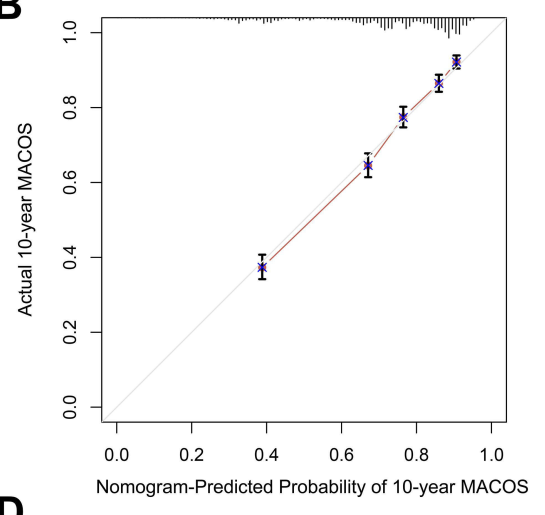

D

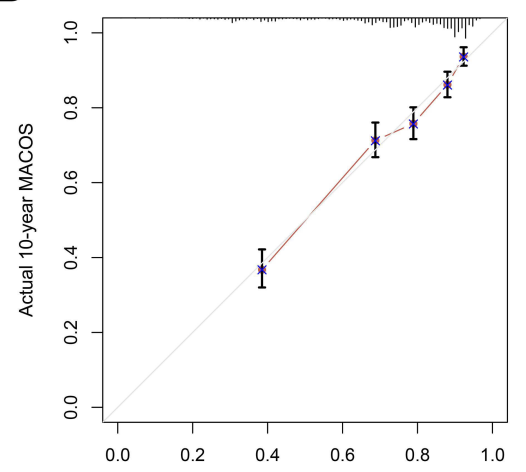

F Nomogram-Predicted Probability of 10-year MACOS

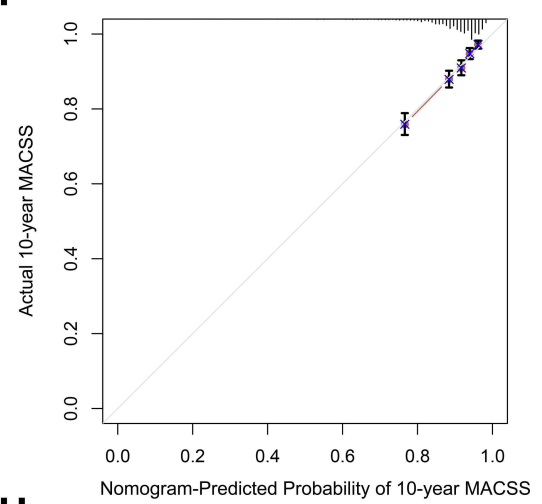

H

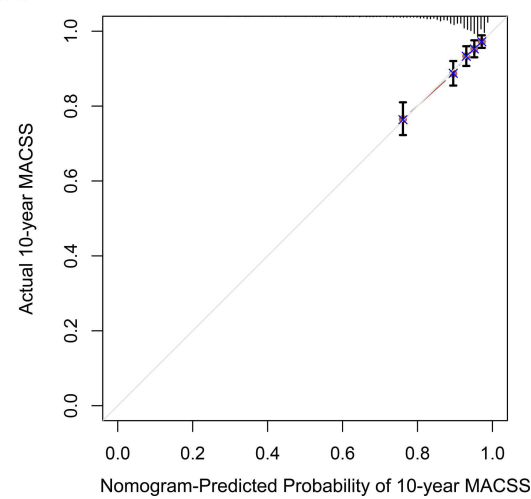

Figure 7 ROC curves and calibration plots of the nomograms for MA patients with ST $\geq 5 Y$,

Notes: ROC curves for predicting 10-year OS and CSS of MA patients with ST $\geq 5 Y$ in the training cohort (A and E) and validation cohort (C and $\mathbf{G})$. Calibration plots of the nomogram prediction of 10-year OS and CSS in the training cohort (B and F) and validation cohort (D and $\mathbf{H})$. The diagonal line shows equality between the actual and predicted survival probability.

Abbreviations: MA, mucinous adenocarcinoma; OS, overall survival; CSS, cancer-specific survival; ROC, receiver operating characteristic curve; AUC, areas under the ROC curve; ST, survival time; Y, year. 
assessment and individualized strategies for cancer management. For some patients who survived for over 5 years, it is recommended to increase the frequency of follow-up.

Age was a stable prognostic factor, independently associated with adverse prognosis for MACSS in patients with $\mathrm{ST}<5 \mathrm{Y}$ or $\mathrm{ST} \geq 5 \mathrm{Y}$. Possible reasons may be that young patients tend to tolerate more intensive therapy and show excellent compliance during the follow-up. ${ }^{17}$ It was also noteworthy that young patients had more advanced stage tumors, higher prevalence of signet ring cell histology, and young age was not associated with improving SRCCSS. ${ }^{18}$ Considering the increasing incidence of early-onset CRC in high-income countries including the USA, ${ }^{5,19}$ this highlights the importance of reconsidering screening guidelines to facilitate early cancer diagnosis and prevent more loss of years of life in these patients.

The pathological TNM stage remains the most important predictor for stratifying risk of mortality in MA and SRCC patients over time in our study. The proportion of patients with more advanced tumor stage was lower among those who survived for over 5 years, and MA and SRCC patients with advanced stage disease had worse survival outcomes in both the $\mathrm{ST}<5 \mathrm{Y}$ and $\mathrm{ST} \geq 5 \mathrm{Y}$ groups. Especially in SRCC patients with $\mathrm{ST} \geq 5 \mathrm{Y}$, TNM stage seemed to be the only prognostic factor associated with CSS. This may further confirm that SRCC histology is highly malignant and represents an independent prognostic factor in CRC patients. Besides, the higher frequency of advanced stage tumor in SRCC than in MA patients may be one of the reasons for the poor prognosis of SRCC. ${ }^{16}$

The frequency of MA and SRCC in the right colon was much higher than that in the left colon and the rectum. ${ }^{20}$ Our results evidenced changes in the prognostic value of primary tumor location with prolonged survival time. In patients with $\mathrm{ST}<5 \mathrm{Y}$, no difference was observed in the HR for MACSS between right-colon, left-colon and rectum location. However, in MA patients with $\mathrm{ST} \geq 5 \mathrm{Y}$, left-side colon and rectum cancer were both associated with an unfavorable prognosis for CSS compared with right-side colon tumors. Different from previous reports, ${ }^{21-23}$ left-sided colon cancer was considered to be related to worse prognosis for MACSS in patients with ST $\geq 5 \mathrm{Y} .{ }^{24}$ Conversely, primary tumor location in rectum was an unfavorable prognostic factor in SRCC patients with ST $<5 \mathrm{Y}$ but had no impact in patients with $\mathrm{ST} \geq 5 \mathrm{Y}$. Previous studies also reported stage-dependent and genotype-dependent differences in outcome between right colon, left colon, and rectum tumors. ${ }^{20,25,26}$ Therefore, the reasons for the distinct effects of primary tumor location on survival outcome in our study remain to be explored.

Tumor size and differentiation grade were identified as independent prognostic factors for MA and SRCC in previous studies. ${ }^{27}$ We also observed that patients with poor tumor differentiation and larger tumor size had adverse survival outcomes in the $\mathrm{ST}<5 \mathrm{Y}$ group. However, and remarkably, neither tumor size nor differentiation grade influenced survival outcome after 5 years of follow-up. Besides, we observed an association between higher frequency of elevated CEA and adverse prognosis of SRCCSS in patients with $\mathrm{ST}<5 \mathrm{Y}$, but this relationship weakened over time in patients with $\mathrm{ST} \geq 5 \mathrm{Y}$. Therefore, it seemed appropriate to re-evaluate the long-term prognosis, eg the 10-year survival probability, in CRC patients who survived for over 5 years.

Accordingly, we constructed novel nomograms specifically for patients who had survived for over 5 years. The nomograms included distinct and stable prognostic factors such as age, TNM stage, CEA level, surgery, and lymph nodes dissection, identified by multivariate Cox regression. Metastasis and older age were, respectively, the parameters accounting for the largest contribution to the CCS and OS nomogram scores. The novel nomograms demonstrated good discrimination and agreement between predictions and observations and should allow both clinicians and patients to intuitively calculate individual 10-year predicted survival probabilities. In addition, based on the interquartile scores from the nomograms, low-, medium-, and high-risk groups were identified, which may help clinicians provide rational suggestions to high-risk patients for additional individualized therapy and intensive follow-up. Although several nomograms have been developed in previous studies, these were mainly established based on parameters related to early death and did not consider potential changes in the impact of prognostic factors over time. ${ }^{28-30}$ Hence, these nomograms may not be appropriate for predicting long-term survival in patients with $\mathrm{ST} \geq 5 \mathrm{Y}$. With the extension of follow-up time, it is important not only to dynamically assess patients' survival outcomes, but also to dynamically adapt prognostic nomograms to specific time frames.

As for treatment options, surgery is the foundation of curative treatment in CRC and our results reconfirmed the positive role of surgery and regional lymph node dissection on MACSS in both the ST<5Y and $\mathrm{ST} \geq 5 \mathrm{Y}$ groups. In 
addition, our analysis showed that SRCC patients could also benefit from surgery of primary site during the initial 5-year follow-up. On the contrary, the receipt of surgery had no association with improving SRCCOS and SRCCSS in patients with a longer survival time. The reasons for high surgical failure rate for SRCC may be the higher proportion of peritoneal or local metastases and a higher rate of circumferential resection margin (CRM) positivity (19\%) in SRCC patients. ${ }^{31,32}$ Multivariable Cox regression analysis showed that radiotherapy had no effect on the prognosis of MA and SRCC patients. However, because few mucinous CRC patients in the SEER database have received radiotherapy, these estimates should be interpreted with caution. Large-scale clinical studies are thus required to assess the prognostic value of different radiotherapy strategies. ${ }^{11}$

Chemotherapy, including the FOLFOX-4, XELOX, FOLFIRI, and 5-FU regimens, is considered as an important and regular treatment option for mucinous CRC patients. ${ }^{11}$ Clinical studies concluded that mucinous CRC patients had a shorter median OS than non-mucinous CRC patients despite improved prognosis resulting from different chemotherapy regimens and the use of adjuvant chemotherapy after surgery. ${ }^{33-35}$ In our study, although chemotherapy was an independent factor associated with favorable prognosis of OS and CSS in MA and SRCC patients with ST $<5 \mathrm{Y}$, its survival benefit weakened over time. ${ }^{36,37}$ In patients with a survival time longer than 5 years, the receipt of chemotherapy was only associated with improving MAOS, but not MACSS, SRCCOS, or SRCCSS. Results of multivariate Cox analyses also showed that the receipt of chemotherapy was independently correlated with $35.8 \%$ decreased risk of overall mortality compared with the non-chemotherapy group in MA patients with $\mathrm{ST}<5 \mathrm{Y}$, and this number fell to $19.9 \%$ in MA patients with $\mathrm{ST} \geq 5 \mathrm{Y}$. Previous studies reported that the survival benefit of adjuvant chemotherapy is time dependent and delayed chemotherapy may be associated with poor survival, especially CSS. ${ }^{38-40}$ Additional chemotherapy-related adverse events due to delayed chemotherapy may be one of the reasons for the poor survival benefit of chemotherapy in patients with $\mathrm{ST} \geq 5 \mathrm{Y} .{ }^{36}$ Moreover, due to the lack of detailed chemotherapy regimen recording and an estimated $10 \%$ rate of under-ascertainment of adjuvant chemotherapy use in the SEER database, ${ }^{41}$ estimates of the survival benefit of chemotherapy should be interpreted with caution and need to be further explored. In summary, some particular CRC patients are still required to receive chemotherapy to improve 5-year survival, but it is necessary for clinicians to reevaluate the prognosis and redefine individual treatment strategies once patients survive for over 5 years.

This study had some inevitable limitations. First, although large, well established, and standardized populations in the SEER database were used for the analyses, the heterogeneous population and the biases inherent to the retrospective nature of the data were the main limitations of our study. Second, several important prognostic factors, such as microsatellite instability, nutritional index, presence of key oncogenic mutations affecting KRAS, NRAS, and BRAF, and detailed information on use of adjuvant chemotherapy and targeted molecular therapy were not recorded in the SEER database. Likewise, tumor recurrence information, which might contribute to further exploration of the prognosis of mucinous CRC, was also lacking. Yet another shortcoming was an irregular recording of the use of surgery, chemotherapy, and radiotherapy in some patients, making it uncertain whether they actually received these treatments. Finally, in our study we did not perform external validation of our predictive model in a cohort from a different database. Although the nomograms performed well in the internal training and validation cohorts from the SEER database, external data would provide more convincing evidence for relatively strict validation. Thus, these biases may lead to an underestimation of the actual prognostic value of our models and require a cautious interpretation of our results.

\section{Conclusion}

In summary, TNM stage was a stable and independent prognostic factor for both MA and SRCC patients who survived for over 5 years. On the contrary, the changes in prognostic factors including tumor size, tumor differentiation, tumor location, surgery, and chemotherapy, highlighted the necessity of dynamic prognosis assessment and personalized followup managements. Therefore, based on prognostic factors identified in the multivariate Cox models, we constructed novel nomograms to visually predict the 10-year OS and CSS probability, which are especially appropriate for MA patients who survived for over 5 years. The novel nomograms demonstrated good accuracy and reliability in prediction by internal validation of discrimination and calibration, as well as clinical utility. Of note, the nomograms may contribute to distinguishing high-risk patients and optimizing the postoperative follow-up regime. This should help further prolong patient survival, especially for early-onset patients who have survived for over 5 years. Furthermore, decreased benefit of 
chemotherapy indicated the urgent need to develop efficient individualized treatment programs for mucinous CRC patients who have survived for over 5 years.

\section{Data Sharing Statement}

The data sets used and/or analyzed during the current study are available from the SEER database and can be accessed through this link: https://seer.cancer.gov/.

\section{Ethical Statements}

This study was approved by the Second Affiliated Hospital of Harbin Medical University and Zhejiang Cancer Hospital. The data were collected from an available public database and no ethical approval was therefore sought for this study. The study used anonymized data and adhered to World Medical Association's Declaration of Helsinki for Ethical Human Research. Informed consent was not required as personal identifying information was not included.

\section{Acknowledgments}

The authors acknowledge the efforts of the Surveillance, Epidemiology, and End Results (SEER) Program tumor registries in the creation of the SEER database.

\section{Author Contributions}

All authors made a significant contribution to this work, whether that is in the conception, study design, execution, acquisition of data, analysis and interpretation, or in all these areas; took part in drafting, revising, or critically reviewing the article; gave final approval of the version to be published; have agreed on the journal to which the article has been submitted; and agree to be accountable for all aspects of the work.

\section{Funding}

This study was supported by the National Natural Science Foundation of China, Grant/Award Number: 81672100; the Key Laboratory for Laboratory Medicine of Jiangsu Province of China, Grant/Award Number: ZDXKB2016005; and the Jiangsu Provincial Commission of Health and Family Planning, Grant/Award Number: H201609.

\section{Disclosure}

The authors report no conflicts of interest in this work.

\section{References}

1. Sung H, Ferlay J, Siegel RL, et al. Global cancer statistics 2020: GLOBOCAN estimates of incidence and mortality worldwide for 36 cancers in 185 countries. CA Cancer J Clin. 2021;71(3):209-249. doi:10.3322/caac.21660

2. Siegel RL, Miller KD, Fuchs HE, Jemal A. Cancer statistics, 2021. CA Cancer J Clin. 2021;71(1):7-33. doi:10.3322/caac.21654

3. Dekker E, Tanis PJ, Vleugels JLA, et al. Colorectal cancer. Lancet. 2019;394(10207):1467-1480. doi:10.1016/S0140-6736(19)32319-0

4. Siegel RL, Miller KD, Goding Sauer A, et al. Colorectal cancer statistics, 2020. CA Cancer J Clin. 2020;70(3):145-164. doi:10.3322/caac.21601

5. Siegel RL, Torre LA, Soerjomataram I, et al. Global patterns and trends in colorectal cancer incidence in young adults. Gut. 2019;68 (12):2179-2185. doi:10.1136/gutjnl-2019-319511

6. Leopoldo S, Lorena B, Cinzia A, et al. Two subtypes of mucinous adenocarcinoma of the colorectum: clinicopathological and genetic features. Ann Surg Oncol. 2008;15(5):1429-1439. doi:10.1245/s10434-007-9757-1

7. Börger ME, Gosens MJ, Jeuken JW, et al. Signet ring cell differentiation in mucinous colorectal carcinoma. J Pathol. 2007;212(3):278-286. doi:10.1002/path.2181

8. Hugen N, van Beek JJ, de Wilt JH, et al. Insight into mucinous colorectal carcinoma: clues from etiology. Ann Surg Oncol. 2014;21(9):2963-2970. doi:10.1245/s10434-014-3706-6

9. Song IH, Hong SM, Yu E, et al. Signet ring cell component predicts aggressive behaviour in colorectal mucinous adenocarcinoma. Pathology. 2019;51(4):384-391. doi:10.1016/j.pathol.2019.03.001

10. Nitsche U, Zimmermann A, Späth C, et al. Mucinous and signet-ring cell colorectal cancers differ from classical adenocarcinomas in tumor biology and prognosis. Ann Surg. 2013;258(5):775-82;discussion 82-3. doi:10.1097/SLA.0b013e3182a69f7e

11. Luo C, Cen S, Ding G, et al. Mucinous colorectal adenocarcinoma: clinical pathology and treatment options. Cancer Commun. 2019;39(1):13. doi:10.1186/s40880-019-0361-0

12. Hyngstrom JR, Hu CY, Xing Y, et al. Clinicopathology and outcomes for mucinous and signet ring colorectal adenocarcinoma: analysis from the National Cancer Data Base. Ann Surg Oncol. 2012;19(9):2814-2821. doi:10.1245/s10434-012-2321-7 
13. Kang H, O'Connell JB, Maggard MA, et al. A 10-year outcomes evaluation of mucinous and signet-ring cell carcinoma of the colon and rectum. Dis Colon Rectum. 2005;48(6):1161-1168. doi:10.1007/s10350-004-0932-1

14. Hugen N, Verhoeven RH, Lemmens VE, et al. Colorectal signet-ring cell carcinoma: benefit from adjuvant chemotherapy but a poor prognostic factor. Int J Cancer. 2015;136(2):333-339. doi:10.1002/ijc.28981

15. Lin JX, Lin JP, Xie JW, et al. Is the AJCC TNM staging system still appropriate for gastric cancer patients survival after 5 years? Eur J Surg Oncol. 2019;45(6):1115-1120. doi:10.1016/j.ejso.2019.01.002

16. Dogrusöz M, Brouwer NJ, de Geus SJR, et al. Prognostic factors five years after enucleation for uveal melanoma. Invest Ophthalmol Vis Sci. 2020;61(3):31. doi:10.1167/iovs.61.3.31

17. Kneuertz PJ, Chang GJ, Hu CY, et al. Overtreatment of young adults with colon cancer: more intense treatments with unmatched survival gains. JAMA Surg. 2015;150(5):402-409. doi:10.1001/jamasurg.2014.3572

18. Wang MJ, Ping J, Li Y, et al. The prognostic factors and multiple biomarkers in young patients with colorectal cancer. Sci Rep. $2015 ; 5: 10645$. doi:10.1038/srep10645

19. Montminy EM, Zhou M, Maniscalco L, et al. Contributions of adenocarcinoma and carcinoid tumors to early-onset colorectal cancer incidence rates in the United States. Ann Intern Med. 2021;174(2):157-166. doi:10.7326/M20-0068

20. Yang J, Du XL, Li ST, et al. Characteristics of differently located colorectal cancers support proximal and distal classification: a population-based study of 57,847 patients. PLoS One. 2016;11(12):e0167540. doi:10.1371/journal.pone.0167540

21. Gao XH, Yu GY, Gong HF, et al. Differences of protein expression profiles, KRAS and BRAF mutation, and prognosis in right-sided colon, left-sided colon and rectal cancer. Sci Rep. 2017;7(1):7882. doi:10.1038/s41598-017-08413-z

22. Petrelli F, Tomasello G, Borgonovo K, et al. Prognostic survival associated with left-sided vs right-sided colon cancer: a systematic review and meta-analysis. JAMA Oncol. 2017;3(2):211-219. doi:10.1001/jamaoncol.2016.4227

23. Zheng C, Jiang F, Lin H, et al. Clinical characteristics and prognosis of different primary tumor location in colorectal cancer: a population-based cohort study. Clin Transl Oncol. 2019;21(11):1524-1531. doi:10.1007/s12094-019-02083-1

24. Qiu MZ, Pan WT, Lin JZ, et al. Comparison of survival between right-sided and left-sided colon cancer in different situations. Cancer Med. 2018;7 (4):1141-1150. doi:10.1002/cam4.1401

25. Weiss JM, Pfau PR, O’Connor ES, et al. Mortality by stage for right- versus left-sided colon cancer: analysis of surveillance, epidemiology, and end results-Medicare data. J Clin Oncol. 2011;29(33):4401-4409. doi:10.1200/JCO.2011.36.4414

26. Xi Y, Yuefen P, Wei W, et al. Analysis of prognosis, genome, microbiome, and microbial metabolome in different sites of colorectal cancer. $J$ Transl Med. 2019;17(1):353. doi:10.1186/s12967-019-2102-1

27. Feng H, Lyu Z, Zheng J, et al. Association of tumor size with prognosis in colon cancer: a Surveillance, Epidemiology, and End Results (SEER) database analysis. Surgery. 2021;169(5):1116-1123. doi:10.1016/j.surg.2020.11.011

28. Liu J, Huang X, Yang W, et al. Nomogram for predicting overall survival in stage II-III colorectal cancer. Cancer Med. 2020;9(7):2363-2371. doi:10.1002/cam4.2896

29. Li Y, Liu W, Zhou Z, et al. Development and validation of prognostic nomograms for early-onset locally advanced colon cancer. Aging. 2020;13 (1):477-492. doi:10.18632/aging.202157

30. Li Y, Liu W, Zhao L, et al. Nomograms predicting overall survival and cancer-specific survival for synchronous colorectal liver-limited metastasis. $J$ Cancer. 2020;11(21):6213-6225. doi:10.7150/jca.46155

31. Fu J, Wu L, Jiang M, et al. Signet ring cell carcinoma of resectable metastatic colorectal cancer has rare surgical value. J Surg Oncol. 2016;114 (8):1004-1008. doi:10.1002/jso.24437

32. Vallam KC, Desouza A, Bal M, Patil P, Engineer R, Saklani A. Adenocarcinoma of the rectum-A composite of three different subtypes with varying outcomes? Clin Colorectal Cancer. 2016;15(2):e47-e52. doi:10.1016/j.clcc.2015.12.004

33. Chand M, Rasheed S, Bhangu A, et al. Adjuvant chemotherapy improves overall survival after TME surgery in mucinous carcinoma of the rectum. Eur J Surg Oncol. 2014;40(2):240-245. doi:10.1016/j.ejso.2013.11.005

34. Hugen N, van de Velde CJ, Bosch SL, et al. Modern treatment of rectal cancer closes the gap between common adenocarcinoma and mucinous carcinoma. Ann Surg Oncol. 2015;22(8):2669-2676. doi:10.1245/s10434-014-4339-5

35. Mekenkamp LJ, Heesterbeek KJ, Koopman M, et al. Mucinous adenocarcinomas: poor prognosis in metastatic colorectal cancer. Eur J Cancer. 2012;48(4):501-509. doi:10.1016/j.ejca.2011.12.004

36. Quénet F, Elias D, Roca L, et al. Cytoreductive surgery plus hyperthermic intraperitoneal chemotherapy versus cytoreductive surgery alone for colorectal peritoneal metastases (PRODIGE 7): a multicentre, randomised, open-label, Phase 3 trial. Lancet Oncol. 2021;22(2):256-266. doi:10.1016/S1470-2045(20)30599-4

37. Gruber-Rouh T, Naguib NN, Eichler K, et al. Transarterial chemoembolization of unresectable systemic chemotherapy-refractory liver metastases from colorectal cancer: long-term results over a 10-year period. Int J Cancer. 2014;134(5):1225-1231. doi:10.1002/ijc.28443

38. Gao P, Huang XZ, Song YX, et al. Impact of timing of adjuvant chemotherapy on survival in stage III colon cancer: a population-based study. $B M C$ Cancer. 2018;18(1):234. doi:10.1186/s12885-018-4138-7

39. Hershman D, Hall MJ, Wang X, et al. Timing of adjuvant chemotherapy initiation after surgery for stage III colon cancer. Cancer. 2006;107 (11):2581-2588. doi:10.1002/cncr.22316

40. Xu F, Rimm AA, Fu P, Krishnamurthi SS, Cooper GS. The impact of delayed chemotherapy on its completion and survival outcomes in stage II colon cancer patients. PLoS One. 2014;9(9):e107993. doi:10.1371/journal.pone.0107993

41. Healy MA, Morris AM, Abrahamse P, Ward KC, Kato I, Veenstra CM. The accuracy of chemotherapy ascertainment among colorectal cancer patients in the surveillance, epidemiology, and end results registry program. BMC Cancer. 2018;18(1):481. doi:10.1186/s12885-018-4405-7 


\section{Publish your work in this journal}

The International Journal of General Medicine is an international, peer-reviewed open-access journal that focuses on general and internal medicine, pathogenesis, epidemiology, diagnosis, monitoring and treatment protocols. The journal is characterized by the rapid reporting of reviews, original research and clinical studies across all disease areas. The manuscript management system is completely online and includes a very quick and fair peer-review system, which is all easy to use. Visit http://www.dovepress.com/testimonials.php to read real quotes from published authors.

Submit your manuscript here: https://www.dovepress.com/international-journal-of-general-medicine-journal 